\title{
COMPUTATION OF PERIODIC SOLUTION BIFURCATIONS IN ODES USING BORDERED SYSTEMS *
}

\author{
E. J. DOEDEL ${ }^{\dagger}$, W. GOVAERTS $\ddagger$, AND YU. A. KUZNETSOV
}

\begin{abstract}
We consider numerical methods for the computation and continuation of the three generic secondary periodic solution bifurcations in autonomous ordinary differential equations (ODEs), namely the fold, the period-doubling (or flip) bifurcation, and the torus (or Neimark-Sacker) bifurcation. In the fold and flip cases we append one scalar equation to the standard periodic boundary value problem (BVP) that defines the periodic solution; in the torus case four scalar equations are appended. Evaluation of these scalar equations and their derivatives requires the solution of linear BVPs, whose sparsity structure (after discretization) is identical to that of the linearization of the periodic BVP. Therefore the calculations can be done using existing numerical linear algebra techniques, such as those implemented in the software AUTO and COLSYS.
\end{abstract}

Key words. bifurcations, periodic solutions, continuation, boundary value problems

AMS subject classifications. 65P30, 65L10, 37M20, 37G15

1. Introduction. We consider parameterized ODEs of the form

$$
\frac{d x}{d t} \equiv x^{\prime}=f(x, \alpha)
$$

where $x \in \mathbf{R}^{n}$ is the state variable, $\alpha \in \mathbf{R}^{m}$ represents parameters, and where $f(x, \alpha) \in \mathbf{R}^{n}$ is a (usually nonlinear) smooth function of $x$ and $\alpha$. Examples of systems of the form (1.1) are ubiquitous in mathematical models in physics, engineering, chemistry, economics, finance, etc.

The simplest solutions of (1.1) are the equilibria, that is, solutions of the equation

$$
f(x, \alpha)=0 .
$$

An equilibrium $\left(x_{0}, \alpha_{0}\right)$ is asymptotically stable if all eigenvalues of the Jacobian matrix $f_{x}\left(x_{0}, \alpha_{0}\right)$ have a strictly negative real part, it is unstable if there is at least one eigenvalue with a strictly positive real part. In generic one-parameter problems, i.e., when $m=1$, eigenvalues on the imaginary axis appear in two ways: as a simple zero eigenvalue, or as a conjugate pair $\pm i \omega, \omega>0$, of purely imaginary eigenvalues. The first case corresponds to a fold, where two solutions coalesce and annihilate each other under parameter variation. The second case corresponds to a Hopf bifurcation, from which periodic solutions emerge. Early papers on the numerical computation of bifurcations of equilibria are [14], [20], and [18].

Periodic solutions are solutions for which $x(T)=x(0)$, for some number $T>0$. The minimal such $T$ is called the period. In generic one-parameter problems, periodic solutions can bifurcate in several ways that can be characterized by the properties of the monodromy matrix. The monodromy matrix is the linearized T-shift along orbits

\footnotetext{
* Submitted to SIAM J. Numer. Anal.

$\dagger^{\dagger}$ Applied Mathematics 217-50, California Institute of Technology, Pasadena CA 91125, USA (on leave from Concordia University, Montreal, Canada); doedelecs.con cordia.ca.

$\ddagger$ Department of Applied Mathematics and Computer Science, University of Gent, Krijgslaan 281S9, B-9000 Gent, Belgium; *illy.govaerts@rug.ac.be.

$\S$ Mathematical Institute, Utrecht University, Budapestlaan 6, P.O. Box 80010, 3508 TA Utrecht, The Netherlands, and Institute of Mathematical Problems of Biology, Russian Academy of Sciences, Pushchino, Moscow Region, 142290 Russia; kuznetsov@math.uu.nl.
} 
of (1.1), evaluated at the point $x(0)$ on the periodic solution. The eigenvalues of this matrix are called the Floquet multipliers of the periodic solution [12], [15].

A periodic solution always has a multiplier equal to 1 . If this multiplier has geometric multiplicity 1 then we call the periodic solution regular. The corresponding eigenvector of the monodromy matrix is the tangent vector to the periodic solution at the point where the monodromy matrix is computed. If all other multipliers are strictly inside the unit circle in the complex plane, then the periodic solution is asymptotically stable. If at least one multiplier has modulus greater than 1 , then the periodic solution is unstable. Three generic bifurcations, determined by the monodromy matrix, can occur along a one-parameter family ("curve" or "branch") of periodic solutions, namely the fold, the period-doubling (or flip) bifurcation, and the torus (or Neimark-Sacker) bifurcation. At a generic fold, the multiplier 1 has algebraic multiplicity 2 and geometric multiplicity 1. Generically, a fold corresponds to a point on the periodic solution branch where the curve turns with respect to the free parameter. At a period-doubling bifurcation there is a simple multiplier equal to -1 . Generically this indicates a period-doubling of the periodic solution, i.e., there are nearby periodic solutions of approximately double period. At a torus bifurcation there is a simple conjugate pair of complex eigenvalues with modulus 1. Generically this corresponds to a bifurcation of an invariant torus, on which the flow contains periodic or quasi-periodic motions.

The aim of this paper is to formulate the computation and continuation of the three generic periodic solution bifurcation curves as minimally extended BVPs, to which standard numerical approximation methods as well as convergence theory apply. Fully extended BVPs for continuing periodic solution bifurcations have been implemented in AUTo [5] (see also [6], [13]). The latter approach doubles the number of function components in the case of the period-doubling and fold bifurcations, and triples it in the case of the torus bifurcation. Fully extended BVPs also yield a more complicated Jacobian sparsity structure (after discretization) than that corresponding to the underlying periodic BVP. There are efficient solution techniques for such sparse linear systems; see, for example, [9]. However, these are not very easy to implement and they are specific for each bifurcation. By contrast, the minimal BVPs proposed in this paper for the period-doubling and fold bifurcations have the same number of function components as the periodic solution problem. In the torus case the number of BVP function components is only doubled. The most important numerical advantage is that only one type of sparse system needs to be solved, namely that corresponding to the underlying periodic BVP. Conceptually, the approach used in this paper is similar to the bordering technique for equilibrium bifurcations [11], $[15]$.

The paper is organized as follows. Section 2 is devoted to the computation and continuation of one-parameter branches of periodic solutions to (1.1). Classical results on regularity of BVPs defining the branches of periodic solutions are proven here for completeness. Sections 3 and 4 present the main results of the paper. Here we construct functionals that vanish at points of bifurcations of the periodic solutions and we prove that they are well-defined and regular. Section 5 deals with various computational issues, including efficient computation of the defining systems and their derivatives. A numerical example is given in Section 6 .

2. Computation and continuation of periodic solutions. Numerical continuation is a technique to compute solution curves to an underdetermined system of equations. Details can be found in, for example, [1], [3], and [11]. It is a basic ingre- 
dient of the numerical bifurcation algorithms implemented in AUTO [5] and CONTENT [16]. In this paper we restrict our discussion to issues that are specific to the case of periodic orbits.

To compute a periodic solution of period $T$ of (1.1), one first fixes the period by rescaling time. Then (1.1) becomes

$$
x^{\prime}(t)=T f(x(t), \alpha)
$$

and we look for solutions of period 1, that is,

$$
x(0)=x(1) .
$$

The period $T$ is one of the unknowns of the problem. In a continuation context we assume that a solution $\left(x_{k-1}(\cdot), T_{k-1}, \alpha_{k-1}\right)$ is known, and we want to find $\left(x_{k}(\cdot), T_{k}, \alpha_{k}\right)$ that we denote by $(x(\cdot), T, \alpha)$. The equations (2.1) and (2.2) together do not fix the solution completely, since any solution can be translated freely in time, that is, if $x(t)$ is a solution then so is $x(t+\sigma)$ for any $\sigma$. To fix the solution it is necessary to add a "phase condition". In AUTO [5] and CONTENT [16] the integral constraint

$$
\int_{0}^{1} x^{*}(\tau) x_{k-1}^{\prime}(\tau) d \tau=0
$$

is used to fix the phase. (We use “*” to denote transpose.)

The periodic solution is now determined by the equations (2.1), (2.2), (2.3), which together form a boundary value problem with an integral constraint.

In our continuation context, the periodic orbit $x(t)$ and the scalars $T$ and $\alpha$ vary along the solution branch. In the setting of Keller's pseudo-arclength continuation method [14] the continuation equation is

$$
\int_{0}^{1}\left(x(\tau)-x_{k-1}(\tau)\right)^{*} \dot{x}_{k-1}(\tau) d \tau+\left(T-T_{k-1}\right) \dot{T}_{k-1}+\left(\alpha-\alpha_{k-1}\right) \dot{\alpha}_{k-1}=\Delta s
$$

where the derivatives are taken with respect to arclength in the function space, and should not be confused with the time derivatives in, for example, Equation (2.3).

A widely used method to discretize the above boundary value problem is the method of orthogonal collocation with piecewise polynomials. It is used in coLsYs [2], as well as in AUTo and ConTEnT. The method is known for its high accuracy [4], and it is particularly suitable for difficult problems, due to its known optimal mesh adaptation techniques [19]. The numerical continuation of the discretized equations leads to structured, sparse linear systems [8]. To describe these systems it is convenient to formulate the boundary value problem in terms of operators on function spaces.

Denote by $\mathcal{C}^{k}\left([a, b], \mathbf{R}^{n}\right)$ the space of $k$ times continuously differentiable functions defined on $[a, b]$ and with values in $\mathbf{R}^{n}$. Let $D$ be the differentiation operator acting from $\mathcal{C}^{1}\left([a, b], \mathbf{R}^{n}\right)$ to $\mathcal{C}^{0}\left([a, b], \mathbf{R}^{n}\right)$. Any $n \times n$ matrix $M(t)$ smoothly depending on $t \in[a, b]$ defines an operator from $\mathcal{C}^{1}\left([a, b], \mathbf{R}^{n}\right)$ into itself by the matrix multiplication: $(M \psi)(t)=M(t) \psi(t)$. The Dirac evaluation operator at the point $t$ is denoted $\delta_{t}$.

For a given $\phi \in \mathcal{C}^{0}\left([0,1], \mathbf{R}^{n}\right)$ we denote by $\operatorname{Int}_{\phi}$ the linear functional from $\mathcal{C}^{0}\left([0,1], \mathbf{R}^{n}\right)$ into $\mathbf{R}$ defined by

$$
\operatorname{Int}_{\phi}(v)=\langle\phi, v\rangle=\int_{0}^{1} \phi^{*}(\tau) v(\tau) d \tau \text {. }
$$


Suppose we want to compute a periodic solution of (1.1), i.e., we want to solve the system $(2.1),(2.2),(2.3)$ and $(2.4)$ for $(x(t), T, \alpha)$ by a Newton-like method. The Fréchet derivative operator corresponding to this problem has the form

$$
\left(\begin{array}{ccc}
D-T f_{x}(x(t), \alpha) & -f(x(t), \alpha) & -T f_{\alpha}(x(t), \alpha) \\
\delta_{0}-\delta_{1} & 0 & 0 \\
\operatorname{Int}_{x_{k-1}^{\prime}}(\cdot) & 0 & 0 \\
\operatorname{Int}_{\dot{x}_{k-1}()} & \dot{T}_{k-1} & \dot{\alpha}_{k-1}
\end{array}\right)
$$

The discrete version of these linear operators is a square matrix that has a large matrix corresponding to $D-T f_{x}(x(t), \alpha)$ in the upper left corner, bordered on the right by two extra columns and at the bottom by $n+2$ extra rows. The big matrix in the upper left corner is a block band matrix. Systems of this form are solved in AUTO by a specially adapted elimination algorithm that computes the multipliers as a byproduct [8].

Consider the fundamental variational equation

$$
X^{\prime}-T f_{x}(x(t), \alpha) X=0
$$

and the adjoint equation

$$
X^{\prime}+T f_{x}^{*}(x(t), \alpha) X=0 .
$$

Denote by $\Phi(t)$ the fundamental matrix solution to (2.6), for which $\Phi(0)=I$, where $I=I_{n \times n}$ is the $n$-dimensional identity matrix. Then $\Phi(1)$ is the monodromy matrix of the periodic solution. The eigenvalues of $\Phi(1)$ are the Floquet multipliers, and there is always at least one multiplier that is equal to 1 . A corresponding eigenvector is $x^{\prime}(0)$. For a regular periodic solution the multiplier 1 has geometric multiplicity 1 . Similarly denote by $\Psi(t)$ the fundamental matrix solution to $(2.7)$, for which $\Psi(0)=I$. One has $\Psi(t)=\left[(\Phi(t))^{-1}\right]^{*}$.

If $v(t)$ is a vector solution to (2.6) with initial values $v(0)=v_{0}$ and $w(t)$ is a vector solution to $(2.7)$ with initial values $w(0)=w_{0}$ then the inner product $w^{*}(t) v(t)=w_{0}^{*} v_{0}$ is independent of time $t$.

The left and right eigenvectors of the monodromy matrix $\Phi(1)$ for a geometrically simple eigenvalue 1 will be denoted $p_{0}, q_{0}$ respectively. It is easily seen that $p_{0}$ (respectively, $q_{0}$ ) is also the right (respectively, left) eigenvector of $\Psi(1)$ for the eigenvalue 1. Furthermore, $q_{0}$ is a scalar multiple of $x^{\prime}(0)$.

We now state some basic facts about the linear operator (2.5) when linearized about a regular periodic solution $(x(t), T, \alpha)$.

Proposition 1. If $(x(t), T, \alpha)$ is a regular periodic solution of (2.1) then the operator

$$
\left[\begin{array}{c}
D-T f_{x}(x(t), \alpha) \\
\delta_{1}-\delta_{0}
\end{array}\right]: \mathcal{C}^{1}\left([0,1], \mathbf{R}^{n}\right) \rightarrow \mathcal{C}^{0}\left([0,1], \mathbf{R}^{n}\right) \times \mathbf{R}^{n}
$$

has a one-dimensional kernel spanned by $\Phi q_{0}$. Its range has codimension 1 ; if $\zeta \in$ $\mathcal{C}^{0}\left([0,1], \mathbf{R}^{n}\right), r \in \mathbf{R}^{n}$ then $(\zeta, r)^{*}$ is in the range if and only if $\left\langle\Psi p_{0}, \zeta\right\rangle=p_{0}^{*} r$. In particular, if $r=0$ then $(\zeta, 0)^{*}$ is in the range if and only if $\left\langle\Psi p_{0}, \zeta\right\rangle=0$.

Proof. First, let $v(t)$ be in the kernel of (2.8). Then $v$ must have the form $v(t)=\Phi(t) v_{0}$ for a vector $v_{0}$. Since $0=\left(\delta_{1}-\delta_{0}\right) v=v(1)-v(0)=(\Phi(1)-I) v_{0}$, we infer that $v_{0}$ must be a right eigenvector of $\Phi(1)$ for the eigenvalue 1 . 
Next, let $\zeta \in \mathcal{C}^{0}\left([0,1], \mathbf{R}^{n}\right), r \in \mathbf{R}^{n}$ be given. If $(\zeta, r)^{*}$ is in the range of (2.8) then there must exist a $v \in \mathcal{C}^{1}\left([0,1], \mathbf{R}^{n}\right)$ for which

$$
v^{\prime}(t)-T f_{x}(x(t), \alpha) v(t)=\zeta(t) .
$$

The general solution of this linear differential equation is

$$
v(t)=\Phi(t)\left[v_{0}+\int_{0}^{t} \Psi^{*}(\tau) \zeta(\tau) d \tau\right]
$$

where $v_{0}=v(0)$ is an initial vector. Also, we must have $v(1)-v(0)=r$, that is,

$$
(\Phi(1)-I) v_{0}+\Phi(1) \int_{0}^{1} \Psi^{*}(\tau) \zeta(\tau) d \tau=r .
$$

Such a vector $v_{0}$ can be found if and only if

$$
p_{0}^{*}\left(\Phi(1) \int_{0}^{1} \Psi^{*}(\tau) \zeta(\tau) d \tau-r\right)=0
$$

that is, if

$$
p_{0}^{*} \int_{0}^{1} \Psi^{*}(\tau) \zeta(\tau) d \tau-p_{0}^{*} r=0
$$

from which the second result follows. $\diamond$

Corollary 1. If $(x(t), T, \alpha)$ is a regular periodic solution of $(2.1)$ then the operator

$$
\left[\begin{array}{c}
D-T f_{x}(x(t), \alpha) \\
\delta_{1}-\delta_{0} \\
\operatorname{Int}_{\phi}
\end{array}\right]
$$

from $\mathcal{C}^{1}\left([0,1], \mathbf{R}^{n}\right)$ into $\mathcal{C}^{0}\left([0,1], \mathbf{R}^{n}\right) \times \mathbf{R}^{n} \times \mathbf{R}$ is one-to-one if and only if $\left\langle\phi, \Phi q_{0}\right\rangle \neq$ 0 .

PRoposition 2. If $(x(t), T, \alpha)$ is a regular periodic solution of $(2.1)$ then the operator

$$
\left[\begin{array}{c}
D+T f_{x}^{*}(x(t), \alpha) \\
\delta_{1}-\delta_{0}
\end{array}\right]: \mathcal{C}^{1}\left([0,1], \mathbf{R}^{n}\right) \rightarrow \mathcal{C}^{0}\left([0,1], \mathbf{R}^{n}\right) \times \mathbf{R}^{n}
$$

has a one-dimensional kernel spanned by $\Psi p_{0}$. Its range has codimension 1 ; if $\zeta \in$ $\mathcal{C}^{0}\left([0,1], \mathbf{R}^{n}\right), r \in \mathbf{R}^{n}$ then $(\zeta, r)^{*}$ is in the range if and only if $\left\langle\Phi q_{0}, \zeta\right\rangle=q_{0}^{*} r$. In particular, if $r=0$ then $(\zeta, 0)^{*}$ is in the range if and only if $\left\langle\Phi q_{0}, \zeta\right\rangle=0$.

Proof. Similar to the proof of Proposition 1. $\diamond$

Corollary 2. If $(x(t), T, \alpha)$ is a regular periodic solution of $(2.1)$ then the operator

$$
\left[\begin{array}{c}
D+T f_{x}^{*}(x(t), \alpha) \\
\delta_{1}-\delta_{0} \\
\operatorname{Int}_{\psi}
\end{array}\right]
$$

from $\mathcal{C}^{1}\left([0,1], \mathbf{R}^{n}\right)$ into $\mathcal{C}^{0}\left([0,1], \mathbf{R}^{n}\right) \times \mathbf{R}^{n} \times \mathbf{R}$ is one-to-one if and only if $\left\langle\psi, \Psi p_{0}\right\rangle \neq$ 0 . 
Proposition 3. Let $(x(t), T, \alpha)$ be a regular periodic solution of $(2.1)$, and let $\phi_{0}, \psi_{0} \in \mathcal{C}^{0}\left([0,1], \mathbf{R}^{n}\right)$ be such that $\left\langle\phi_{0}, \Phi q_{0}\right\rangle \neq 0,\left\langle\psi_{0}, \Psi p_{0}\right\rangle \neq 0$. Then the operator

$$
\left[\begin{array}{cc}
D-T f_{x}(x(t), \alpha) & \psi_{0} \\
\delta_{1}-\delta_{0} & 0 \\
\operatorname{Int}_{\phi_{0}} & 0
\end{array}\right]
$$

from $\mathcal{C}^{1}\left([0,1], \mathbf{R}^{n}\right) \times \mathbf{R}$ into $\mathcal{C}^{0}\left([0,1], \mathbf{R}^{n}\right) \times \mathbf{R}^{n} \times \mathbf{R}$ is one-to-one and onto.

Proof. To prove that the operator is one-to-one, suppose that

$$
\left[\begin{array}{cc}
D-T f_{x}(x(t), \alpha) & \psi_{0} \\
\delta_{1}-\delta_{0} & 0 \\
\operatorname{Int}_{\phi_{0}} & 0
\end{array}\right]\left(\begin{array}{c}
v \\
G
\end{array}\right)=\left(\begin{array}{l}
0 \\
0 \\
0
\end{array}\right),
$$

for $v \in \mathcal{C}^{1}\left([0,1], \mathbf{R}^{n}\right), G \in \mathbf{R}$. In particular, it follows that

$$
\left[\begin{array}{c}
D-T f_{x}(x(t), \alpha) \\
\delta_{0}-\delta_{1}
\end{array}\right] v=\left(\begin{array}{c}
-G \psi_{0} \\
0
\end{array}\right) .
$$

Since $\left\langle\psi_{0}, \Psi p_{0}\right\rangle \neq 0$, it follows from the last statement in Proposition 1 that $G=0$. By Corollary 1 and the assumption that $\left\langle\phi_{0}, \Phi q_{0}\right\rangle \neq 0$, it follows that $v=0$ as well.

To prove that the operator is onto we consider the equation

$$
\left[\begin{array}{cc}
D-T f_{x}(x(t), \alpha) & \psi_{0} \\
\delta_{1}-\delta_{0} & 0 \\
\operatorname{Int}_{\phi_{0}} & 0
\end{array}\right]\left(\begin{array}{c}
v \\
G
\end{array}\right)=\left(\begin{array}{c}
\zeta \\
r \\
s
\end{array}\right),
$$

where $\zeta \in \mathcal{C}^{0}\left([0,1], \mathbf{R}^{n}\right), r \in \mathbf{R}^{n}, s \in \mathbf{R}$. In particular, the first two equations can be written

$$
\left[\begin{array}{c}
D-T f_{x}(x(t), \alpha) \\
\delta_{1}-\delta_{0}
\end{array}\right] v=\left(\begin{array}{c}
\zeta-G \psi_{0} \\
r
\end{array}\right)
$$

By Proposition 1 this equation is solvable for $v$, say, $v=v_{p}$, if

$$
\left\langle\Psi p_{0}, \zeta-G \psi_{0}\right\rangle=p_{0}^{*} r
$$

that is, if we choose

$$
G=G_{p} \equiv \frac{\left\langle\Psi p_{0}, \zeta\right\rangle-p_{0}^{*} r}{\left\langle\Psi p_{0}, \psi_{0}\right\rangle}
$$

where, by assumption, the denominator does not vanish. Now

$$
v(t)=v_{p}(t)+c \Phi(t) q_{0}
$$

is also a solution of (2.14), for any constant $c$. The third equation in (2.13) can now be written as

$$
\int_{0}^{1} \phi_{0}^{*}(\tau)\left[v_{p}(\tau)+c \Phi(\tau) q_{0}\right] d \tau=s .
$$

By the assumption that $\left\langle\phi_{0}, \Phi q_{0}\right\rangle \neq 0$ it follows that the third equation is satisfied if we take

$$
c=\frac{s-\int_{0}^{1} \phi_{0}^{*}(\tau) v_{p}(\tau) d \tau}{\int_{0}^{1} \phi_{0}^{*}(\tau) \Phi(\tau) q_{0} d \tau} \cdot \diamond
$$


Proposition 4. Let $(x(t), T, \alpha)$ be a regular periodic solution of $(2.1)$, and let $\phi_{0}, \psi_{0} \in \mathcal{C}^{0}\left([0,1], \mathbf{R}^{n}\right)$ be such that $\left\langle\phi_{0}, \Phi q_{0}\right\rangle \neq 0,\left\langle\psi_{0}, \Psi p_{0}\right\rangle \neq 0$. Then the operator

$$
\left[\begin{array}{cc}
D+T f_{x}^{*}(x(t), \alpha) & \phi_{0} \\
\delta_{1}-\delta_{0} & 0 \\
\operatorname{Int}_{\psi_{0}} & 0
\end{array}\right]
$$

from $\mathcal{C}^{1}\left([0,1], \mathbf{R}^{n}\right) \times \mathbf{R}$ into $\mathcal{C}^{0}\left([0,1], \mathbf{R}^{n}\right) \times \mathbf{R}^{n} \times \mathbf{R}$ is one-to-one and onto.

Proof. Similar to the proof of Proposition $3 . \diamond$

3. Test functionals for bifurcations of periodic solutions. For the fold and Hopf bifurcations of equilibria, several minimally extended systems are discussed in [11] and incorporated in CONTENT [10].

To obtain similar systems in the case of periodic orbits we construct functionals that vanish at codimension- 1 bifurcations of periodic solutions, i.e., at the fold (limit point), at the period-doubling (flip), and at the torus bifurcation, respectively.

3.1. A test functional for the fold bifurcation. Let $(x(t), T, \alpha)$ define a periodic solution of (1.1), i.e., it satisfies (2.1), (2.2) and (2.3). If the solution corresponds to a fold then the monodromy matrix $\Phi(1)$ has an eigenvalue +1 with algebraic multiplicity 2 and geometric multiplicity 1. (A geometrically double eigenvalue +1 corresponds to a higher degeneracy.)

Let $p_{0}$ and $q_{0}$ denote the corresponding left and right eigenvectors, which satisfy

$$
\begin{aligned}
(\Phi(1)-I) q_{0}=0, & (\Psi(1)-I) p_{0}=0, \\
(\Phi(1)-I)^{*} p_{0}=0, & (\Psi(1)-I)^{*} q_{0}=0 .
\end{aligned}
$$

with

$$
p_{0}^{*} p_{0}=q_{0}^{*} q_{0}=1
$$

At a generic fold, where the multiplier 1 has algebraic multiplicity 2, we also have generalized eigenvectors $p_{1}$ and $q_{1}$ satisfying

$$
(\Phi(1)-I) q_{1}=q_{0}, \quad(\Psi(1)-I) p_{1}=p_{0},
$$

where $q_{1}$ and $p_{1}$ can be chosen so that

$$
q_{1}^{*} q_{0}=p_{1}^{*} p_{0}=0
$$

Note that in the multiplicity-2 case we also have $p_{0}^{*} q_{0}=p_{1}^{*}(\Psi(1)-I)^{*} q_{0}=0$.

Proposition 5. If $(x(t), T, \alpha)$ is a regular periodic solution of $(2.1)$ then the operator

$$
\left[\begin{array}{cc}
D-T f_{x}(x(t), \alpha) & -f(x(t), \alpha) \\
\delta_{1}-\delta_{0} & 0 \\
\operatorname{Int}_{f(x(\cdot), \alpha)} & 0
\end{array}\right]
$$

from $\mathcal{C}^{1}\left([0,1], \mathbf{R}^{n}\right) \times \mathbf{R} \rightarrow \mathcal{C}^{0}\left([0,1], \mathbf{R}^{n}\right) \times \mathbf{R}^{n} \times \mathbf{R}$ is one-to-one if the multiplier 1 has algebraic multiplicity 1 . If the multiplier 1 has algebraic multiplicity 2 , i.e., at a fold, then the operator has a one-dimensional kernel, spanned by the vector

$$
\left(\begin{array}{c}
v \\
1
\end{array}\right) \in \mathcal{C}^{1}\left([0,1], \mathbf{R}^{n}\right) \times \mathbf{R},
$$


where $v(t)=\frac{c_{0}}{T} \Phi(t)\left(c_{2} q_{0}-\left(q_{1}-t q_{0}\right)\right), c_{2}$ is determined by the condition that

$$
q_{0}^{*} \int_{0}^{1} \Phi^{*}(\tau) \Phi(\tau)\left[c_{2} q_{0}-\left(q_{1}-\tau q_{0}\right)\right] d \tau=0,
$$

and $c_{0}$ is determined by the condition that $x^{\prime}(0)=c_{0} q_{0}$.

Proof. Consider the homogeneous equations

$$
\left[\begin{array}{cc}
D-T f_{x}(x(t), \alpha) & -f(x(t), \alpha) \\
\delta_{1}-\delta_{0} & 0 \\
\operatorname{Int}_{f(x(\cdot), \alpha)} & 0
\end{array}\right]\left(\begin{array}{l}
v \\
S
\end{array}\right)=\left(\begin{array}{l}
0 \\
0 \\
0
\end{array}\right) .
$$

From the first equation in (3.3) we have

$$
\begin{aligned}
v(t)=\Phi(t)\left[v_{0}+S \int_{0}^{t} \Psi^{*}(\tau) f(x(\tau), \alpha) d \tau\right] & =\Phi(t)\left[v_{0}+\frac{S}{T} \int_{0}^{t} \Psi^{*}(\tau) x^{\prime}(\tau) d \tau\right] \\
& =\Phi(t)\left[v_{0}+\frac{S}{T} \int_{0}^{t} \Psi^{*}(\tau) \Phi(\tau) d \tau x^{\prime}(0)\right]=\Phi(t)\left[v_{0}+\frac{S t}{T} x^{\prime}(0)\right]
\end{aligned}
$$

where we used the facts that $\Psi^{*}(\tau) \Phi(\tau)=I$ and $x^{\prime}(t)=\Phi(t) x^{\prime}(0)$. Above, $v_{0}=v(0)$ is an initial vector. By the second equation in (3.3) we have

$$
0=v(1)-v(0)=(\Phi(1)-I) v_{0}+\frac{S}{T} x^{\prime}(0),
$$

that is,

$$
(\Phi(1)-I) v_{0}=-\frac{S}{T} x^{\prime}(0)
$$

Now $(\Phi(1)-I) x^{\prime}(0)=0$, so that $x^{\prime}(0)=c_{0} q_{0}$, for some $c_{0} \in \mathbf{R}, c_{0} \neq 0$. Thus we must solve

$$
(\Phi(1)-I) v_{0}=-c_{0} \frac{S}{T} q_{0},
$$

where $q_{0}$ spans the kernel of $\Phi(1)-I$.

If the multiplier 1 has algebraic multiplicity 1 then we must have $S=0, v_{0}=c_{1} q_{0}$, and hence $v(t)=c_{1} \Phi(t) q_{0}$. By the third equation in (3.3)

$$
0=\int_{0}^{1} f^{*}(x(\tau), \alpha) v(\tau) d \tau=\frac{1}{T} \int_{0}^{1} x^{\prime *}(\tau) v(\tau) d \tau=\frac{1}{T} \int_{0}^{1}\left[\Phi(\tau) x^{\prime}(0)\right]^{*} c_{1} \Phi(\tau) q_{0} d \tau,
$$

or

$$
c_{0} c_{1} q_{0}^{*}\left(\int_{0}^{1} \Phi^{*}(\tau) \Phi(\tau) d \tau\right) q_{0}=0
$$

from which it follows that $c_{1}=0$. Thus $v(t) \equiv 0$. It follows that the operator (3.1) is one-to-one.

At a fold the multiplier 1 has algebraic multiplicity 2. In this case (3.4) is also solvable if $S$ is nonzero, namely

$$
v_{0}=-c_{0} \frac{S}{T} q_{1}+c_{2} q_{0},
$$


where $c_{2} \in \mathbf{R}$ is arbitrary. The third equation in (3.3) then implies

$$
\begin{aligned}
0 & =\int_{0}^{1} x^{* *}(\tau) v(\tau) d \tau \\
& =\int_{0}^{1} x^{*}(\tau) \Phi(\tau)\left[v_{0}+\frac{S \tau}{T} x^{\prime}(0)\right] d \tau \\
& =\int_{0}^{1} x^{*}(\tau) \Phi(\tau)\left[-c_{0} \frac{S}{T} q_{1}+c_{2} q_{0}+\frac{S \tau}{T} c_{0} q_{0}\right] d \tau \\
& =\int_{0}^{1}\left[\Phi(\tau) x^{\prime}(0)\right]^{*} \Phi(\tau)\left[-c_{0} \frac{S}{T} q_{1}+c_{2} q_{0}+\frac{S \tau}{T} c_{0} q_{0}\right] d \tau \\
& =c_{0} q_{0}^{*} \int_{0}^{1} \Phi^{*}(\tau) \Phi(\tau)\left[-c_{0} \frac{S}{T} q_{1}+c_{2} q_{0}+\frac{S \tau}{T} c_{0} q_{0}\right] d \tau
\end{aligned}
$$

from which it follows that

$$
c_{2}=\frac{c_{0} S q_{0}^{*} \int_{0}^{1} \Phi^{*}(\tau) \Phi(\tau)\left[q_{1}-\tau q_{0}\right] d \tau}{T q_{0}^{*} \int_{0}^{1} \Phi^{*}(\tau) \Phi(\tau) d \tau q_{0}} .
$$

Proposition 6. Let $(x(t), T, \alpha)$ be a regular periodic solution of $(2.1)$ and consider the operator

$$
M_{1}=\left[\begin{array}{cc}
D-T f_{x}(x(t), \alpha) & -f(x(t), \alpha) \\
\delta_{1}-\delta_{0} & 0 \\
\operatorname{Int}_{f(x(\cdot), \alpha)} & 0
\end{array}\right]
$$

from $\mathcal{C}^{1}\left([0,1], \mathbf{R}^{n}\right) \times \mathbf{R} \rightarrow \mathcal{C}^{0}\left([0,1], \mathbf{R}^{n}\right) \times \mathbf{R}^{n} \times \mathbf{R}$. If the multiplier 1 has algebraic multiplicity one, then $M_{1}$ is onto. If it has algebraic multiplicity two, i.e., at a fold, then the range of $M_{1}$ has codimension 1 and the vector

$$
\left(\begin{array}{c}
\Psi p_{0} \\
-p_{0} \\
0
\end{array}\right) \in \mathcal{C}^{0}\left([0,1], \mathbf{R}^{n}\right) \times \mathbf{R}^{n} \times \mathbf{R}
$$

is complementary to the range space.

Proof. Consider a vector $(\xi, \eta, \omega)^{*}$ in $\mathcal{C}^{0}\left([0,1], \mathbf{R}^{n}\right) \times \mathbf{R}^{n} \times \mathbf{R}$. This vector is in the range of $M_{1}$ if and only if there exist $(v, S)^{*}$ in $\mathcal{C}^{1}\left([0,1], \mathbf{R}^{n}\right) \times \mathbf{R}$ such that

$$
M_{1}\left(\begin{array}{c}
v \\
S
\end{array}\right)=\left(\begin{array}{c}
\xi \\
\eta \\
\omega
\end{array}\right)
$$

The first equation in (3.7) implies that

$$
v(t)=\Phi(t)\left[v(0)+\int_{0}^{t} \Psi^{*}(\tau)(\xi(\tau)+S f(x(\tau), \alpha)) d \tau\right]
$$

The second equation in (3.7) then implies

$$
\eta=v(1)-v(0)=(\Phi(1)-I) v(0)+\Phi(1) \int_{0}^{1} \Psi^{*}(\tau)(\xi(\tau)+S f(x(\tau), \alpha)) d \tau
$$

Now

$$
\int_{0}^{1} \Psi^{*}(\tau) f(x(\tau), \alpha) d \tau=\frac{1}{T} \int_{0}^{1} \Psi^{*}(\tau) x^{\prime}(\tau) d \tau=\frac{1}{T} \int_{0}^{1} \Psi^{*}(\tau) c_{0} \Phi(\tau) q_{0} d \tau=\frac{c_{0}}{T} q_{0}
$$

So

$$
\eta=(\Phi(1)-I) v(0)+\frac{S c_{0}}{T} q_{0}+\Phi(1) \int_{0}^{1} \Psi^{*}(\tau) \xi(\tau) d \tau
$$


If 1 is an algebraically simple eigenvalue of $\Phi(1)$ then $q_{0}$ is not in the range of $(\Phi(1)-I)$. For given $\xi, \eta(3.8)$ can be solved for $v(0), S$. Moreover, the solution is unique up to the addition of a scalar multiple of $q_{0}$ to $v(0)$. Since

$$
\int_{0}^{1}\left(x^{\prime}(\tau)\right)^{*} \Phi(\tau) q_{0} d \tau=c_{0} \int_{0}^{1}\left(\Phi(\tau) q_{0}\right)^{*} \Phi(\tau) q_{0} d \tau \neq 0
$$

the scalar is determined uniquely by the third equation in (3.7).

If 1 is an algebraically double eigenvalue of $\Phi(1)$, i.e., at a fold point, then (3.8) is solvable if and only if

$$
p_{0}^{*} \eta=p_{0}^{*} \int_{0}^{1} \Psi^{*}(\tau) \xi(\tau) d \tau
$$

If so, the third equation in (3.7) again determines the solution uniquely. $\diamond$

Proposition 7 . If $(x(t), T, \alpha)$ is a regular periodic solution of $(2.1)$ then the operator

$$
\left[\begin{array}{cc}
D+T f_{x}^{*}(x(t), \alpha) & -f(x(t), \alpha) \\
\delta_{1}-\delta_{0} & 0 \\
\operatorname{Int}_{f(x(\cdot), \alpha)} & 0
\end{array}\right]
$$

from $\mathcal{C}^{1}\left([0,1], \mathbf{R}^{n}\right) \times \mathbf{R} \rightarrow \mathcal{C}^{0}\left([0,1], \mathbf{R}^{n}\right) \times \mathbf{R}^{n} \times \mathbf{R}$ is one-to-one if the multiplier 1 has algebraic multiplicity 1 . If the multiplier 1 has algebraic multiplicity 2, i.e., at a fold, then the operator has a one-dimensional kernel, spanned by $\left(\Psi^{*} p_{0}, 0\right)^{*} \in$ $\mathcal{C}^{1}\left([0,1], \mathbf{R}^{n}\right) \times \mathbf{R}$.

Proof. Consider the homogeneous equations

$$
\left[\begin{array}{cc}
D+T f_{x}^{*}(x(t), \alpha) & -f(x(t), \alpha) \\
\delta_{1}-\delta_{0} & 0 \\
\operatorname{Int}_{f(x(), \alpha)} & 0
\end{array}\right]\left(\begin{array}{c}
w \\
R
\end{array}\right)=\left(\begin{array}{l}
0 \\
0 \\
0
\end{array}\right)
$$

From the first equation in (3.10) we have

$$
w(t)=\Psi(t)\left[w_{0}+\frac{R}{T} \int_{0}^{t} \Phi^{*}(\tau) x^{\prime}(\tau) d \tau\right]
$$

where $w_{0}=w(0)$ is an initial vector. The second equation in (3.10) implies

$$
0=w(1)-w(0)=(\Psi(1)-I) w_{0}+\frac{R}{T} \Psi(1) \int_{0}^{1} \Phi^{*}(\tau) x^{\prime}(\tau) d \tau
$$

or

$$
(\Psi(1)-I) w_{0}=-\frac{R}{T} \Psi(1) \int_{0}^{1} \Phi^{*}(\tau) \Phi(\tau) d \tau x^{\prime}(0) .
$$

Given $R$, this equation is solvable for $w_{0}$ if

$$
-R q_{0}^{*} \Psi(1) \int_{0}^{1} \Phi^{*}(\tau) \Phi(\tau) d \tau x^{\prime}(0)=0,
$$

that is, recalling that $x^{\prime}(0)=c_{0} q_{0}, c_{0} \neq 0$, and $q_{0}^{*} \Psi(1)=q_{0}^{*}$, if

$$
c_{0} R q_{0}^{*} \int_{0}^{1} \Phi^{*}(\tau) \Phi(\tau) d \tau q_{0}=0 .
$$


It follows that $R=0$, independently of the algebraic multiplicity of the eigenvalue 1 . Thus $w(t)=\Psi(t) w_{0}$, where $(\Psi(1)-I) w_{0}=0$, so that $w_{0}=c_{3} p_{0}$, for some $c_{3} \in \mathbf{R}$.

From the third equation in (3.10) it follows that

$$
\begin{aligned}
0 & =\int_{0}^{1} w^{*}(\tau) x^{\prime}(\tau) d \tau=\int_{0}^{1}\left[c_{3} \Psi(\tau) p_{0}\right]^{*} \Phi(\tau) x^{\prime}(0) d \tau \\
& =c_{0} c_{3} p_{0}^{*} \int_{0}^{1} \Psi^{*}(\tau) \Phi(\tau) d \tau q_{0}=c_{0} c_{3} p_{0}^{*} q_{0} .
\end{aligned}
$$

If the multiplier 1 has algebraic multiplicity 1 then $p_{0}^{*} q_{0} \neq 0$. In this case $c_{3}=0$ and hence $w(t) \equiv 0$, that is, the operator (3.9) is one-to-one.

If the multiplier 1 has algebraic multiplicity 2 then $p_{0}^{*} q_{0}=0$, and we can choose $c_{3} \neq 0$. In this case $w_{0} \neq 0$, hence $w(t) \not \equiv 0$. It follows that the operator (3.9) has a one-dimensional kernel. $\diamond$

PRoposition 8. If $(x(t), T, \alpha)$ is a regular periodic solution of (2.1) then the operator

$$
M_{2}=\left[\begin{array}{cc}
D+T f_{x}^{*}(x(t), \alpha) & -f(x(t), \alpha) \\
\delta_{1}-\delta_{0} & 0 \\
\operatorname{Int}_{f(x(\cdot), \alpha)} & 0
\end{array}\right]
$$

from $\mathcal{C}^{1}\left([0,1], \mathbf{R}^{n}\right) \times \mathbf{R} \rightarrow \mathcal{C}^{0}\left([0,1], \mathbf{R}^{n}\right) \times \mathbf{R}^{n} \times \mathbf{R}$ is onto if the multiplier 1 has algebraic multiplicity one. If the multiplier 1 has algebraic multiplicity two, i.e., at a fold, then its range has codimension 1 and the vector $(0,0,1)^{*} \in \mathcal{C}^{0}\left([0,1], \mathbf{R}^{n}\right) \times \mathbf{R}^{n} \times \mathbf{R}$ is complementary to the range space.

Proof. Consider a vector $(\xi, \eta, \omega)^{*}$ in $\mathcal{C}^{0}\left([0,1], \mathbf{R}^{n}\right) \times \mathbf{R}^{n} \times \mathbf{R}$. This vector is in the range of $M_{2}$ if and only if there exist $(w, R)^{*}$ in $\mathcal{C}^{1}\left([0,1], \mathbf{R}^{n}\right) \times \mathbf{R}$ such that

$$
M_{2}\left(\begin{array}{c}
w \\
R
\end{array}\right)=\left(\begin{array}{c}
\xi \\
\eta \\
\omega
\end{array}\right)
$$

The first equation in (3.12) implies that

$$
w(t)=\Psi(t)\left[w(0)+\int_{0}^{t} \Phi^{*}(\tau)\left(\xi(\tau)+R c_{0} \Phi(\tau) q_{0}\right) d \tau\right]
$$

The second equation in (3.12) then implies

$$
\eta=w(1)-w(0)=(\Psi(1)-I) w(0)+\Psi(1) \int_{0}^{1} \Phi^{*}(\tau)\left(\xi(\tau)+R c_{0} \Phi(\tau) q_{0}\right) d \tau
$$

We so obtain the equation

$$
(\Psi(1)-I) w(0)=\eta-R c_{0} \Psi(1) \int_{0}^{1} \Phi^{*}(\tau) \Phi(\tau) q_{0} d \tau-\Psi(1) \int_{0}^{1} \Phi^{*}(\tau) \xi(\tau) d \tau .
$$

This equation for $w(0)$ is solvable if and only if

$$
q_{0}^{*} \eta=R c_{0} q_{0}^{*} \int_{0}^{1} \Phi^{*}(\tau) \Phi(\tau) d \tau+q_{0}^{*} \int_{0}^{1} \Phi^{*}(\tau) \xi(\tau) d \tau
$$


The latter equation is solvable uniquely for $R$, so the previous one is solvable for $w(0)$ and defines it up to the addition of a scalar multiple of $p_{0}$.

Now suppose that $(w, R)^{*}$ solve the first two equations in $(3.12)$ where $w(0)=$ $w_{0}+r p_{0}$ and $r$ is arbitrary. The third equation in (3.12) then requires

$$
q_{0}^{*}\left(w_{0}+r p_{0}\right) d t=\omega T .
$$

If the eigenvalue 1 of $\Phi(1)$ has algebraic multiplicity 1 , then this equation has a unique solution in $r$ and so $M_{2}$ is one-to-one and onto. If it has algebraic multiplicity 2, then the range of $M_{2}$ has codimension at most 1 . In this case, for $\xi=0, \eta=0, \omega=1$ we have $w_{0}=0$ and (3.13) has no solution. So the range of $M_{2}$ has codimension 1 and $(0,0,1)^{*}$ is a vector complementary to that range. $\diamond$

Proposition 9. Let $(x(t), T, \alpha)$ be a regular periodic solution of $(2.1)$ where $\Phi(1)$ has eigenvalue 1 with algebraic multiplicity 2 . Then there exist $v_{01}, w_{01}, v_{11}, w_{11}$ $\in \mathcal{C}^{0}\left([0,1], \mathbf{R}^{n}\right), w_{02}, v_{12} \in \mathbf{R}^{n}, w_{03}, v_{02}, v_{13}, w_{12} \in \mathbf{R}$ such that

$$
N_{1}=\left[\begin{array}{ccc}
D-T f_{x}(x(t), \alpha) & -f(x(t), \alpha) & w_{01} \\
\delta_{1}-\delta_{0} & 0 & w_{02} \\
\operatorname{Int}_{f(x(\cdot), \alpha)} & 0 & w_{03} \\
\operatorname{Int}_{v_{01}} & v_{02} & 0
\end{array}\right]
$$

and

$$
N_{2}=\left[\begin{array}{ccc}
D+T f_{x}^{*}(x(t), \alpha) & -f(x(\cdot), \alpha) & v_{11} \\
\delta_{1}-\delta_{0} & 0 & v_{12} \\
\operatorname{Int}_{f(x(\cdot), \alpha)} & 0 & v_{13} \\
\operatorname{Int}_{w_{11}} & w_{12} & 0
\end{array}\right]
$$

from $\mathcal{C}^{1}\left([0,1], \mathbf{R}^{n}\right) \times \mathbf{R}^{n} \times \mathbf{R}$ to $\mathcal{C}^{0}\left([0,1], \mathbf{R}^{n}\right) \times \mathbf{R}^{n} \times \mathbf{R} \times \mathbf{R}$ are one-to-one and onto

For any such choice of the bordering elements we define $v, w \in \mathcal{C}^{1}\left([0,1], \mathbf{R}^{n}\right)$ and $S, G, H, R \in \mathbf{R}$ by the equations

$$
N_{1}\left(\begin{array}{c}
v \\
S \\
G
\end{array}\right)=\left(\begin{array}{l}
0 \\
0 \\
0 \\
1
\end{array}\right)
$$

and

$$
N_{2}\left(\begin{array}{c}
w \\
R \\
H
\end{array}\right)=\left(\begin{array}{c}
0 \\
0 \\
0 \\
-1
\end{array}\right)
$$

Then in a neighborhood of $(x(t), T, \alpha), G=0$ if and only if $H=0$. Moreover, this happens if and only if the regular periodic solution corresponds to a fold bifurcation.

Proof. We choose

$$
\left(\begin{array}{c}
v_{01}(t) \\
v_{02}
\end{array}\right)=\left(\begin{array}{c}
v(t) \\
1
\end{array}\right),
$$

where $v$ is given in the statement of Proposition 5. Further we set

$$
\left(\begin{array}{c}
w_{01}(t) \\
w_{02} \\
w_{03}
\end{array}\right)=\left(\begin{array}{c}
\Psi^{*}(t) p_{0} \\
0 \\
0
\end{array}\right)
$$


By Propositions 5 and $6 N_{1}$ is one-to-one and onto. We further set

$$
\begin{gathered}
\left(\begin{array}{c}
w_{11}(t) \\
w_{12}
\end{array}\right)=\left(\begin{array}{c}
\Psi^{*}(t) p_{0} \\
0
\end{array}\right) \\
\left(\begin{array}{c}
v_{11}(t) \\
v_{12} \\
v_{13}
\end{array}\right)=\left(\begin{array}{l}
0 \\
0 \\
1
\end{array}\right) .
\end{gathered}
$$

By Propositions 7 and $8 N_{2}$ is one-to-one and onto. The last statement in the Proposition is proved by standard arguments. $\diamond$

3.2. A test functional for the period-doubling bifurcation. At a generic period-doubling bifurcation there is an algebraically simple Floquet multiplier equal to -1 . The left and right eigenvectors of the monodromy matrix $\Phi(1)$ for the eigenvalue -1 will be denoted by $p_{2}$ and $q_{2}$, respectively. They are also the right and left eigenvector, respectively, of $\Psi(1)$ for the eigenvalue -1 .

Proposition 10. If $(x(t), T, \alpha)$ corresponds to a period-doubling bifurcation then the operator

$$
\left[\begin{array}{c}
D-T f_{x}(x(t), \alpha) \\
\delta_{0}+\delta_{1}
\end{array}\right]: \mathcal{C}^{1}\left([0,1], \mathbf{R}^{n}\right) \rightarrow \mathcal{C}^{0}\left([0,1], \mathbf{R}^{n}\right) \times \mathbf{R}^{n}
$$

has a one-dimensional kernel spanned by $\Phi q_{2}$. Its range has codimension 1 ; if $\zeta \in$ $\mathcal{C}^{0}\left([0,1], \mathbf{R}^{n}\right), r \in \mathbf{R}^{n}$ then $(\zeta, r)^{*}$ is in the range if and only if $\left\langle\Psi p_{2}, \zeta\right\rangle=-p_{2}^{*} r$. In particular, if $r=0$ then $(\zeta, 0)^{*}$ is in the range if and only if $\left\langle\Psi p_{2}, \zeta\right\rangle=0$.

Proof. Similar to the proof of Proposition $1 . \diamond$

Corollary 3 . If $(x(t), T, \alpha)$ corresponds to a period-doubling bifurcation then the operator

$$
\left[\begin{array}{c}
D-T f_{x}(x(t), \alpha) \\
\delta_{0}+\delta_{1} \\
\operatorname{Int}_{\phi}
\end{array}\right]
$$

from $\mathcal{C}^{1}\left([0,1], \mathbf{R}^{n}\right)$ into $\mathcal{C}^{0}\left([0,1], \mathbf{R}^{n}\right) \times \mathbf{R}^{n} \times \mathbf{R}$ is one-to-one if and only if $\left\langle\phi, \Phi q_{2}\right\rangle \neq$ 0 .

Proposition 11. If $(x(t), T, \alpha)$ corresponds to a period-doubling bifurcation then the operator

$$
\left[\begin{array}{c}
D+T f_{x}^{*}(x(t), \alpha) \\
\delta_{0}+\delta_{1}
\end{array}\right]: \mathcal{C}^{1}\left([0,1], \mathbf{R}^{n}\right) \rightarrow \mathcal{C}^{0}\left([0,1], \mathbf{R}^{n}\right) \times \mathbf{R}^{n}
$$

has a one-dimensional kernel spanned by $\Psi p_{2}$. Its range has codimension 1 ; if $\zeta \in$ $\mathcal{C}^{0}\left([0,1], \mathbf{R}^{n}\right), r \in \mathbf{R}^{n}$ then $(\zeta, r)^{*}$ is in the range if and only if $\left\langle\Phi q_{2}, \zeta\right\rangle=-q_{2}^{*} r$. In particular, if $r=0$ then $(\zeta, 0)^{*}$ is in the range if and only if $\left\langle\Phi q_{2}, \zeta\right\rangle=0$.

Proof. Similar to the proof of Proposition 2. $\diamond$

Corollary 4. If $(x(t), T, \alpha)$ corresponds to a period-doubling bifurcation then the operator

$$
\left[\begin{array}{c}
D+T f_{x}^{*}(x(t), \alpha) \\
\delta_{0}+\delta_{1} \\
\operatorname{Int}_{\psi}
\end{array}\right]
$$


from $\mathcal{C}^{1}\left([0,1], \mathbf{R}^{n}\right)$ into $\mathcal{C}^{0}\left([0,1], \mathbf{R}^{n}\right) \times \mathbf{R}^{n} \times \mathbf{R}$ is one-to-one if and only if $\left\langle\psi, \Psi p_{2}\right\rangle \neq$ 0 .

Proposition 12. Let $(x(t), T, \alpha)$ correspond to a period-doubling bifurcation and let $\phi_{0}, \psi_{0} \in \mathcal{C}^{0}\left([0,1], \mathbf{R}^{n}\right)$ be such that $\left\langle\phi_{0}, \Phi q_{2}\right\rangle \neq 0,\left\langle\psi_{0}, \Psi p_{2}\right\rangle \neq 0$. Then the operator

$$
\left[\begin{array}{cc}
D-T f_{x}(x(t), \alpha) & \psi_{0} \\
\delta_{0}+\delta_{1} & 0 \\
\operatorname{Int}_{\phi_{0}} & 0
\end{array}\right]
$$

from $\mathcal{C}^{1}\left([0,1], \mathbf{R}^{n}\right) \times \mathbf{R}$ into $\mathcal{C}^{0}\left([0,1], \mathbf{R}^{n}\right) \times \mathbf{R}^{n} \times \mathbf{R}$ is one-to-one and onto.

Proof. Similar to the proof of Proposition 3.

Proposition 13. Let $(x(t), T, \alpha)$ correspond to a period-doubling bifurcation and let $\phi_{0}, \psi_{0} \in \mathcal{C}^{0}\left([0,1], \mathbf{R}^{n}\right)$ be such that $\left\langle\phi_{0}, \Phi q_{2}\right\rangle \neq 0,\left\langle\psi_{0}, \Psi p_{2}\right\rangle \neq 0$. Then the operator

$$
\left[\begin{array}{cc}
D+T f_{x}^{*}(x(t), \alpha) & \phi_{0} \\
\delta_{0}+\delta_{1} & 0 \\
\operatorname{Int}_{\psi_{0}} & 0
\end{array}\right]
$$

from $\mathcal{C}^{1}\left([0,1], \mathbf{R}^{n}\right) \times \mathbf{R}$ into $\mathcal{C}^{0}\left([0,1], \mathbf{R}^{n}\right) \times \mathbf{R}^{n} \times \mathbf{R}$ is one-to-one and onto.

Proof. Similar to the proof of Proposition $4 . \vee$

Proposition 14. Let $(x(t), T, \alpha)$ be a periodic solution close to a period-doubling bifurcation, so that the operators $M_{3}$ and $M_{4}$ (defined below) from $\mathcal{C}^{1}\left([0,1], \mathbf{R}^{n}\right) \times \mathbf{R}$ into $\mathcal{C}^{0}\left([0,1], \mathbf{R}^{n}\right) \times \mathbf{R}^{n} \times \mathbf{R}$, are both one-to-one and onto. Let $v, w \in \mathcal{C}^{1}\left([0,1], \mathbf{R}^{n}\right), G$, $H \in \mathbf{R}$ be defined by the equations

$$
\begin{gathered}
M_{3}\left(\begin{array}{c}
v \\
G
\end{array}\right) \equiv\left[\begin{array}{cc}
D-T f_{x}(x(t), \alpha) & \psi_{0} \\
\delta_{0}+\delta_{1} & 0 \\
\operatorname{Int}_{\phi_{0}} & 0
\end{array}\right]\left(\begin{array}{c}
v \\
G
\end{array}\right)=\left(\begin{array}{c}
0 \\
0 \\
1
\end{array}\right), \\
M_{4}\left(\begin{array}{c}
w \\
H
\end{array}\right) \equiv\left[\begin{array}{cc}
D+T f_{x}^{*}(x(t), \alpha) & \phi_{0} \\
\delta_{0}+\delta_{1} & 0 \\
\operatorname{Int}_{\psi_{0}} & 0
\end{array}\right]\left(\begin{array}{c}
w \\
H
\end{array}\right)=\left(\begin{array}{c}
0 \\
0 \\
-1
\end{array}\right) .
\end{gathered}
$$

Then $G=H$. Furthermore, $G=0$ if and only if the periodic solution corresponds to a period-doubling bifurcation. If so, then $v(0)$ is the right eigenvector of the monodromy matrix for the eigenvalue -1 .

Proof. Multiplying the first equation in (3.22) on the left with $w^{*}(t)$, integrating over the interval $[0,1]$, and using the last equation in $(3.23)$ we obtain

$$
\int_{0}^{1} w^{*} v^{\prime}(\tau) d \tau-T \int_{0}^{1} w^{*}(\tau) f_{x}(x(\tau), \alpha) v(\tau) d \tau-G=0
$$

Partial integration of the first term, using the second equations in (3.22) and (3.23), gives

$$
-\int_{0}^{1} v^{*}(\tau) w^{\prime}(\tau) d \tau-T \int_{0}^{1} v^{*}(\tau) f_{x}^{*}(x(\tau), \alpha) w(\tau) d \tau-G=0
$$

Using the first equation in (3.23) we get

$$
-\left\langle v,\left(-H \phi_{0}\right)\right\rangle-G=0
$$

Using the third equation in (3.22) we obtain $G=H$. The other statements in the Proposition are now obvious. $\diamond$ 
3.3. A test functional for the torus bifurcation. Let $e^{ \pm i \theta}, 0<\theta<\pi$ be the conjugate pair of complex multipliers with modulus 1 . Furthermore, let $p_{1}, p_{2} \in \mathbf{R}^{n}$ (respectively, $q_{1}, q_{2} \in \mathbf{R}^{n}$ ) be such that $p_{1}+i p_{2}$ (respectively, $q_{1}+i q_{2}$ ) is a left (respectively, right) complex eigenvector of the monodromy matrix $\Phi(1)$. So

$$
\begin{aligned}
\left(p_{1}+i p_{2}\right)^{H} \Phi(1) & =e^{i \theta}\left(p_{1}+i p_{2}\right)^{H}, \\
\Phi(1)\left(q_{1}+i q_{2}\right) & =e^{i \theta}\left(q_{1}+i q_{2}\right), \\
\Psi(1)\left(p_{1}+i p_{2}\right) & =e^{i \theta}\left(p_{1}+i p_{2}\right), \\
\left(q_{1}+i q_{2}\right)^{H} \Psi(1) & =e^{i \theta}\left(q_{1}+i q_{2}\right)^{H},
\end{aligned}
$$

where $\left(p_{1}+i p_{2}\right)^{H}=p_{1}^{*}-i p_{2}^{*},\left(q_{1}+i q_{2}\right)^{H}=q_{1}^{*}-i q_{2}^{*}$.

In this section it is convenient to extend the definition of $x(t), \Phi(t)$, and $\Psi(t)$ to the interval $[0,2]$ by periodicity with period 1 , and to redefine

$$
\operatorname{Int}_{\phi}(v)=\langle\phi, v\rangle=\int_{0}^{2} \phi^{*}(\tau) v(\tau) d \tau .
$$

We start with the following result.

PROPOSITION 15. Let $(x(t), T, \alpha)$ define a periodic solution that is, it satisfies (2.1), (2.2) and (2.3). Let $(x(t), T, \alpha)$ corresponds to a torus bifurcation through multipliers $e^{ \pm i \theta}, 0<\theta<\pi$. Let $\kappa=\cos \theta$ and consider the operator

$$
\left[\begin{array}{c}
D-T f_{x}(x(t), \alpha) \\
\delta_{0}-2 \kappa \delta_{1}+\delta_{2}
\end{array}\right]
$$

from $\mathcal{C}^{1}\left([0,2], \mathbf{R}^{n}\right) \rightarrow \mathcal{C}^{0}\left([0,2], \mathbf{R}^{n}\right) \times \mathbf{R}^{n}$. Then

(i) The operator (3.24) has a two-dimensional kernel spanned by $\Phi(t) q_{1}$ and $\Phi(t) q_{2}$.

(ii) The operator (3.24) has a range with codim 2. The vectors

$$
\left(\begin{array}{c}
\Psi p_{1} \\
0
\end{array}\right),\left(\begin{array}{c}
\Psi p_{2} \\
0
\end{array}\right) \in \mathcal{C}^{0}\left([0,2], \mathbf{R}^{n}\right) \times \mathbf{R}^{n}
$$

span a two-dimensional subspace that is complementary to the range of (3.24).

Proof. Let $v$ be in the kernel of (3.24). Then $v$ must have the form $v(t)=\Phi(t) v_{0}$ with $v_{0} \in \mathbf{R}^{n}$. We further have

$$
0=\left(\delta_{0}-2 \kappa \delta_{1}+\delta_{2}\right) v=v(0)-2 \kappa v(1)+v(2)=\left(\Phi(1)-e^{i \theta} I\right)\left(\Phi(1)-e^{-i \theta} I\right) v_{0} .
$$

We infer that it is necessary and sufficient that $v_{0}$ is in the span of $q_{1}, q_{2}$.

As a first step in the proof of (ii) we consider $\zeta \in \mathcal{C}^{0}\left([0,2], \mathbf{R}^{n}\right), r \in \mathbf{R}^{n}$ and give a necessary and sufficient condition in order that $(\zeta, r)^{*}$ be in the range of (3.24). First, there must exist a $v \in \mathcal{C}^{1}\left([0,2], \mathbf{R}^{n}\right)$ for which

$$
v^{\prime}(t)-T f_{x}(x(t), \alpha) v(t)=\zeta(t) .
$$

The general solution of this linear differential equation is

$$
v(t)=\Phi(t)\left[v_{0}+\int_{0}^{t} \Psi^{*}(\tau) \zeta(\tau) d \tau\right]
$$


where $v_{0}=v(0)$ is an initial vector. Also, we must have $v(0)-2 \kappa v(1)+v(2)=r$, that is,

$\left(\Phi(1)-e^{i \theta} I\right)\left(\Phi(1)-e^{-i \theta} I\right) v_{0}-2 \kappa \Phi(1) \int_{0}^{1} \Psi^{*}(\tau) \zeta(\tau) d \tau+\Phi(1)^{2} \int_{0}^{2} \Psi^{*}(\tau) \zeta(\tau) d \tau=r$.

This is an equation for $v_{0}$ which is solvable if and only if

$$
-2 \kappa p^{H} \Phi(1) \int_{0}^{1} \Psi^{*}(\tau) \zeta(\tau) d \tau+p^{H} \Phi(1)^{2} \int_{0}^{2} \Psi^{*}(\tau) \zeta(\tau) d \tau=p^{H} r
$$

or, equivalently,

$$
-2 \kappa e^{i \theta} \int_{0}^{1} p^{H} \Psi^{*}(\tau) \zeta(\tau) d \tau+e^{2 i \theta} \int_{0}^{2} p^{H} \Psi^{*}(\tau) \zeta(\tau) d \tau=p^{H} r
$$

If we define the linear functional $L$ by setting

$$
L(\zeta)=-2 \kappa e^{i \theta} \int_{0}^{1} p^{H} \Psi^{*}(\tau) \zeta(\tau) d \tau+e^{2 i \theta} \int_{0}^{2} p^{H} \Psi^{*}(\tau) \zeta(\tau) d \tau
$$

then we infer that $(\zeta, r)^{*}$ is in the range of (3.24) if and only if $L(\zeta)=p^{H} r$.

As a second step in the proof of (ii) we compute $L\left(\Psi p_{1}\right)$ and $L\left(\Psi p_{2}\right)$. We have

$$
L\left(\Psi p_{1}\right)=-2 \cos \theta e^{i \theta} \int_{0}^{1} p^{H} \Psi^{*}(\tau) \Psi(\tau) p_{1} d \tau+e^{2 i \theta} \int_{0}^{2} p^{H} \Psi^{*}(\tau) \Psi(\tau) p_{1} d \tau
$$

$=e^{i \theta}(-2 \cos \theta+\cos \theta+i \sin \theta) \int_{0}^{1} p^{H} \Psi^{*}(\tau) \Psi(\tau) d \tau+e^{2 i \theta} \int_{0}^{1} p^{H} \Psi^{*}(1+\tau) \Psi(1+\tau) p_{1} d \tau$.

Now we note that

$$
\Psi(1+\tau) p_{1}=\Psi(\tau) \Psi(1) p_{1}=\Psi(\tau)\left(\cos \theta p_{1}-\sin \theta p_{2}\right)
$$

and

$$
p^{H} \Psi^{*}(1+\tau)=[\Psi(\tau) \Psi(1) p]^{H}=\left[e^{i \theta} \Psi(\tau) p\right]^{H}=e^{-i \theta} p^{H} \Psi^{*}(\tau) .
$$

Hence

$$
L\left(\Psi p_{1}\right)=i \sin \theta e^{i \theta} \int_{0}^{1} p^{H} \Psi^{*}(\tau) \Psi(\tau) p d \tau=(-\sin \theta+i \cos \theta) \sin \theta \int_{0}^{1}\|\Psi(\tau) p\|^{2} d \tau
$$

By a similar argument we find that

$$
L\left(\Psi p_{2}\right)=(\cos \theta+i \sin \theta) \sin \theta \int_{0}^{1}\|\Psi(\tau) p\|^{2} d \tau .
$$

As a third step in the proof of (ii) we show that the range of (3.24) has codimension 2 by proving that every $(\xi, r)^{*}$ can be written in a unique way as

$$
\left(\begin{array}{c}
\xi \\
r
\end{array}\right)=\left(\begin{array}{c}
\xi_{0} \\
r_{0}
\end{array}\right)+\alpha\left(\begin{array}{c}
0 \\
p_{1}
\end{array}\right)+\beta\left(\begin{array}{c}
0 \\
p_{2}
\end{array}\right)
$$


with $\left(\xi_{0}, r_{0}\right)^{*}$ in the range of $(3.24)$ and $\alpha, \beta \in \mathbf{R}$.

Obviously $\xi_{0}=\xi$ and $r_{0}$ has to satisfy the conditions

$$
p^{H} r_{0}=L(\xi), \quad r_{0}=r-\alpha p_{1}-\beta p_{2} .
$$

These conditions imply

$$
\left(\begin{array}{ll}
p_{1}^{*} p_{1} & p_{1}^{*} p_{2} \\
p_{2}^{*} p_{1} & p_{2}^{*} p_{2}
\end{array}\right)\left(\begin{array}{c}
\alpha \\
\beta
\end{array}\right)=\left(\begin{array}{c}
p_{1}^{*} r-\operatorname{Re}[L(\xi)] \\
p_{2}^{*} r+\operatorname{Im}[L(\xi)]
\end{array}\right) .
$$

This nonsingular linear system defines $\alpha, \beta$ in a unique way. Next, $r_{0}$ is obviously defined by the requirement $r_{0}=r-\alpha p_{1}-\beta p_{2}$ and with this choice we have $p^{H} r_{0}=$ $L(\xi)$.

As the fourth and last step to prove (ii) we will show that

$$
\left(\begin{array}{c}
\Psi p_{1} \\
0
\end{array}\right),\left(\begin{array}{c}
\Psi p_{2} \\
0
\end{array}\right)
$$

also span a two-dimensional space complementary to the range of (3.24). To this end we decompose

$$
\begin{aligned}
& \left(\begin{array}{c}
\Psi p_{1} \\
0
\end{array}\right)=\left(\begin{array}{c}
\Psi p_{1} \\
r_{1}
\end{array}\right)+\alpha_{1}\left(\begin{array}{c}
0 \\
p_{1}
\end{array}\right)+\beta_{1}\left(\begin{array}{c}
0 \\
p_{2}
\end{array}\right), \\
& \left(\begin{array}{c}
\Psi p_{2} \\
0
\end{array}\right)=\left(\begin{array}{c}
\Psi p_{2} \\
r_{2}
\end{array}\right)+\alpha_{2}\left(\begin{array}{c}
0 \\
p_{1}
\end{array}\right)+\beta_{2}\left(\begin{array}{c}
0 \\
p_{2}
\end{array}\right) .
\end{aligned}
$$

in the decomposition of (3.26). Then $\alpha_{1}, \beta_{1}, \alpha_{2}, \beta_{2}$ are defined by the matrix equation

$$
\left(\begin{array}{ll}
p_{1}^{*} p_{1} & p_{1}^{*} p_{2} \\
p_{2}^{*} p_{1} & p_{2}^{*} p_{2}
\end{array}\right)\left(\begin{array}{cc}
\alpha_{1} & \alpha_{2} \\
\beta_{1} & \beta_{2}
\end{array}\right)=\left(\begin{array}{rr}
-\operatorname{Re}\left[L\left(\Psi p_{1}\right)\right] & -\operatorname{Re}\left[L\left(\Psi p_{2}\right]\right. \\
\operatorname{Im}\left[L\left(\Psi p_{1}\right)\right] & \operatorname{Im}\left[L\left(\Psi p_{2}\right]\right.
\end{array}\right) .
$$

The proof of (ii) is complete if we show that

$$
\left(\begin{array}{ll}
\alpha_{1} & \alpha_{2} \\
\beta_{1} & \beta_{2}
\end{array}\right)
$$

is a nonsingular matrix, or equivalently that

$$
\left(\begin{array}{rr}
-\operatorname{Re}\left[L\left(\Psi p_{1}\right)\right] & -\operatorname{Re}\left[L\left(\Psi p_{2}\right]\right. \\
\operatorname{Im}\left[L\left(\Psi p_{1}\right)\right] & \operatorname{Im}\left[L\left(\Psi p_{2}\right]\right.
\end{array}\right)
$$

is nonsingular. By the second step this matrix is equal to

$$
\left(\begin{array}{rr}
\sin \theta & -\cos \theta \\
\cos \theta & \sin \theta
\end{array}\right) \sin \theta \int_{0}^{1}\|\Psi(\tau) p\|^{2} d \tau
$$

Since $\sin \theta \neq 0(3.27)$ the proof is complete. $\diamond$

Proposition 16. Let $(x(t), T, \alpha)$ define a periodic solution, that is, it satisfies (2.1), (2.2), and (2.3). Let $(x(t), T, \alpha)$ correspond to a torus bifurcation through multipliers $e^{ \pm i \theta}, 0<\theta<\pi$. Set $\kappa=\cos \theta$ and consider the operator

$$
\left[\begin{array}{c}
D+T f_{x}^{*}(x(t), \alpha) \\
\delta_{0}-2 \kappa \delta_{1}+\delta_{2}
\end{array}\right]
$$


from $\mathcal{C}^{1}\left([0,2], \mathbf{R}^{n}\right) \rightarrow \mathcal{C}^{0}\left([0,2], \mathbf{R}^{n}\right) \times \mathbf{R}^{n}$. Then

(i) The operator (3.28) has a two-dimensional kernel spanned by $\Psi(t) p_{1}$ and $\Psi(t) p_{2}$

(ii) The operator (3.28) has a range with codimension 2. The vectors

$$
\left(\begin{array}{c}
\Phi q_{1} \\
0
\end{array}\right),\left(\begin{array}{c}
\Phi q_{2} \\
0
\end{array}\right) \in \mathcal{C}^{0}\left([0,2], \mathbf{R}^{n}\right) \times \mathbf{R}^{n}
$$

span a two-dimensional subspace that is complementary to the range of (3.28).

Proof. Similar to the proof of the preceding Proposition. $\diamond$

Corollary 5. Let $(x(t), T, \alpha)$ correspond to the torus bifurcation point of a periodic solution. If $\kappa=\cos \theta$ then the operators

$$
\left[\begin{array}{ccc}
D-T f_{x}(x(t), \alpha) & \Psi p_{1} & \Psi p_{2} \\
\delta_{0}-2 \kappa \delta_{1}+\delta_{2} & 0 & 0 \\
\operatorname{Int}_{\Phi(\cdot) q_{1}} & 0 & 0 \\
\operatorname{Int}_{\Phi(\cdot) q_{2}} & 0 & 0
\end{array}\right]
$$

and

$$
\left[\begin{array}{ccc}
D+T f_{x}^{*}(x(t), \alpha) & \Phi q_{1} & \Phi q_{2} \\
\delta_{0}-2 \kappa \delta_{1}+\delta_{2} & 0 & 0 \\
\operatorname{Int}_{\Psi(\cdot) p_{1}} & 0 & 0 \\
\operatorname{Int}_{\Psi(\cdot) p_{2}} & 0 & 0
\end{array}\right]
$$

from $\mathcal{C}^{1}\left([0,2], \mathbf{R}^{n}\right) \times \mathbf{R}^{2}$ into $\mathcal{C}^{0}\left([0,2], \mathbf{R}^{n}\right) \times \mathbf{R}^{n} \times \mathbf{R}^{2}$ are both one-to-one and onto.

Proof. Standard.

Proposition 17. Let $(x(t), T, \alpha)$ be close to a torus bifurcation point of periodic solutions and $\kappa$ close to the value $\cos \theta$ of the torus bifurcation point so that the operators

$$
\begin{aligned}
M_{5}= & {\left[\begin{array}{ccc}
D-T f_{x}(x(t), \alpha) & \psi_{0} & \psi_{1} \\
\delta_{0}-2 \kappa \delta_{1}+\delta_{2} & 0 & 0 \\
\operatorname{Int}_{\phi_{0}} & 0 & 0 \\
\operatorname{Int}_{\phi_{1}} & 0 & 0
\end{array}\right], } \\
M_{6}= & {\left[\begin{array}{ccc}
D+T f_{x}^{*}(x(t), \alpha) & \phi_{0} & \phi_{1} \\
\delta_{0}-2 \kappa \delta_{1}+\delta_{2} & 0 & 0 \\
\operatorname{Int}_{\psi_{0}} & 0 & 0 \\
\operatorname{Int}_{\psi_{1}} & 0 & 0
\end{array}\right] }
\end{aligned}
$$

from $\mathcal{C}^{1}\left([0,2], \mathbf{R}^{n}\right) \times \mathbf{R}^{2}$ into $\mathcal{C}^{0}\left([0,2], \mathbf{R}^{n}\right) \times \mathbf{R}^{n} \times \mathbf{R}^{2}$ are both one-to-one and onto. Let $v_{1}, v_{2}, w_{1}, w_{2} \in \mathcal{C}^{1}\left([0,2], \mathbf{R}^{n}\right), G, H \in \mathbf{R}^{2 \times 2}$ be defined by the equations

$$
\begin{gathered}
M_{5}\left(\begin{array}{cc}
v_{1} & v_{2} \\
G_{11} & G_{12} \\
G_{21} & G_{22}
\end{array}\right)=\left(\begin{array}{cc}
0 & 0 \\
0 & 0 \\
1 & 0 \\
0 & 1
\end{array}\right) \\
M_{6}\left(\begin{array}{cc}
w_{1} & w_{2} \\
H_{11} & H_{21} \\
H_{12} & H_{22}
\end{array}\right)=\left(\begin{array}{cc}
0 & 0 \\
0 & 0 \\
-1 & 0 \\
0 & -1
\end{array}\right)
\end{gathered}
$$


If $(x(t), T, \alpha)$ is a periodic solution, then $G=0$ if and only if $H=0$. Moreover, this happens if and only if $(x(t), T, \alpha)$ corresponds to a torus bifurcation of periodic solutions with the multipliers $e^{ \pm i \theta}$, where $\kappa=\cos (\theta)$.

Proof. Standard. $\diamond$

4. Regularity of the defining systems. In this section we prove that, under natural nondegeneracy and transversality conditions, the test functionals constructed in the previous section are regular (with respect to the arclength parameter along the periodic solution branch). This implies regularity of defining systems consisting of the periodic BVP (2.1), (2.2), (2.3), and the vanishing condition for the corresponding functional, for the two-parameter continuation of the bifurcation.

4.1. Regularity at a fold bifurcation. The prove the regularity of the test functional $G$ for the fold bifurcation in Proposition 9, we proceed as in the case of the fold bifurcation of equilibria [11].

The computation of periodic orbits is based on the equation

$$
F(X, \alpha)=0
$$

where $X \equiv(x(\cdot), T) \in \mathcal{C}^{1}([0,1], \mathbf{R}) \times \mathbf{R}$, and $F(X) \in \mathcal{C}^{0}([0,1], \mathbf{R}) \times \mathbf{R}^{n} \times \mathbf{R}$ is given by

$$
F(X) \equiv\left(\begin{array}{l}
x^{\prime}(t)-T f(x(t), \alpha) \\
x(1)-x(0) \\
\int_{0}^{1} x^{*}(\tau) x_{k-1}^{\prime}(\tau) d \tau
\end{array}\right)
$$

(see $(2.1),(2.2)$, and (2.3)). The Fréchet derivative $F_{X}(X, \alpha)$ of this operator (with $x_{k-1}$ substituted by $x$ upon differentiation) is $M_{1}$ as defined in (3.1). By Propositions 5 and 6 the periodic orbit is a fold point if and only if $F_{X}$ is singular; the left and right singular vectors are then

$$
\left(\begin{array}{c}
\Psi p_{0} \\
-p_{0} \\
0
\end{array}\right)
$$

and

$$
\left(\begin{array}{l}
v \\
1
\end{array}\right)
$$

given in (3.2) and (3.6) respectively. The fold point is regular if and only if

$$
\left(\begin{array}{c}
\Psi p_{0} \\
-p_{0} \\
0
\end{array}\right)^{*} F_{X X}\left(\begin{array}{l}
v \\
1
\end{array}\right)\left(\begin{array}{l}
v \\
1
\end{array}\right) \neq 0
$$

Now let $\alpha$ be a scalar parameter, such that $\left[\begin{array}{ll}F_{X} & F_{\alpha}\end{array}\right]$ is onto at the fold point. Let $s$ denote arclength along the branch of periodic orbits. We think of $X$ and $\alpha$ as functions of $s$ so that (4.1) is an identity in $s$. By (3.14) this also defines $G$ as a function of $s$. Suppose that the fold bifurcation occurs at $s=s_{0}$. We will prove that (4.3) is equivalent to $G_{s}\left(s_{0}\right) \neq 0$.

Taking derivatives of (3.14) with respect to $s$ we find

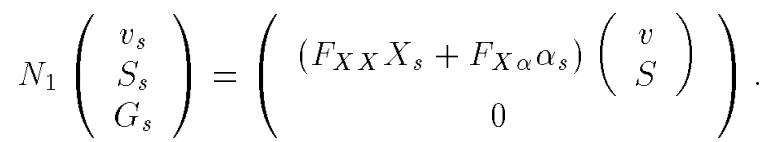


In this expression

$$
\left(\begin{array}{l}
v \\
S
\end{array}\right)
$$

is a right singular vector of $F_{X}$. Furthermore, at the fold point $\alpha_{s}=0$. Since $F_{X} X_{s}+F_{\alpha} \alpha_{s} \equiv 0$ it follows that $X_{s}$ is also a right singular vector of $F_{X}$. Now by (4.4) we have $G_{s}\left(s_{0}\right) \neq 0$ if and only if

$$
F_{X X}\left(\begin{array}{l}
v \\
1
\end{array}\right)\left(\begin{array}{l}
v \\
1
\end{array}\right)
$$

is not in the range of $M_{1}$; this is equivalent to (4.3).

4.2. Regularity at a period-doubling bifurcation. We have seen that locally, near a period-doubling bifurcation, the system consisting of (2.1), (2.2), (2.3), and $G=0$ (where $G$ is given by (3.22)), defines the set of period-doubling bifurcations in $(x(\cdot), T, \alpha)$-space, if the conditions $\left\langle\phi_{0}, \Phi q_{0}\right\rangle \neq 0,\left\langle\psi_{0}, \Psi p_{0}\right\rangle \neq 0$ hold. We will now prove that this is a regular system if the appropriate nondegeneracy and transversality conditions for the period-doubling bifurcation hold.

Let $s$ denote arclength along the curve of periodic orbits so that $(x(s)(t), T(s), \alpha(s))$ is a solution of $(2.1),(2.2)$, and $(2.3)$ for all $s$ near the bifurcation value $s_{0}$. Nondegeneracy implies that -1 is algebraically simple eigenvalue of $\Phi\left(s_{0}\right)(1)$, so that it can be continued smoothly, together with its left and right eigenvectors, for nearby values of $s$. Specifically, we denote by $\lambda(s)$ an eigenvalue of $\Phi(s)(1)$, with left and right eigenvectors $p(s), q(s)$, that is,

$$
\begin{array}{ll}
\Phi(s)(1) q(s)=\lambda(s) q(s), & p^{*}(s) \Phi(s)(1)=\lambda(s) p^{*}(s) \\
\Psi(s)(1) p(s)=\lambda^{-1}(s) p(s), & q^{*}(s) \Psi(s)(1)=\lambda^{-1}(s) q^{*}(s) \\
p\left(s_{0}\right)=p_{0}, & q\left(s_{0}\right)=q_{0} \\
\lambda\left(s_{0}\right)=-1 . &
\end{array}
$$

The nondegeneracy condition implies that

$$
p^{*}(s) q(s) \neq 0
$$

for all $s$ sufficiently close to $s_{0}$. By standard arguments, (4.5) implies

$$
p^{*}(s) q(s) \lambda_{s}(s)=p^{*}(s) \Phi_{s}(s)(1) q(s) .
$$

To get an explicit formula for $\Phi_{s}\left(s_{0}\right)(1)$ we start from the observation that

$$
\left(D-T(s) f_{x}(x(s), \alpha(s))\right) \Phi=0 .
$$

Taking derivatives, and using somewhat simplied notation, we obtain

$$
\left(D-T f_{x}\right) \Phi_{s}=\left(T f_{x}\right)_{s} \Phi .
$$

Multiplying on the right by an arbitrary vector $\xi \in \mathbf{R}^{n}$, we have

$$
\left(D-T f_{x}\right) \Phi_{s} \xi=\left(T f_{x}\right)_{s} \Phi \xi .
$$

This is a linear differential equation for $\Phi_{s} \xi$ with solution

$$
\Phi_{s}(s)(t) \xi=\Phi(s)(t)\left[\zeta+\int_{0}^{t} \Psi^{*}(s)(\tau)\left(T f_{x}\right)_{s}(s)(\tau) \Phi(s)(\tau) \xi d \tau\right]
$$


for some $\zeta \in \mathbf{R}^{n}$. For $t=0$ this reduces to

$$
\Phi_{s}(s)(0) \xi=\Phi(s)(0) \zeta
$$

Since $\Phi(s)(0)=I, \Phi_{s}(s)(0)=0$, this implies that $\zeta=0$, so that

$$
\Phi_{s}(s)(t) \xi=\Phi(s)(t) \int_{0}^{t} \Psi^{*}(s)(\tau)\left(T f_{x}\right)_{s}(s)(\tau) \Phi(s)(\tau) \xi d \tau
$$

for all $\xi \in \mathbf{R}^{n}$. From (4.7) we get

$$
p^{*}(s) q(s) \lambda_{s}(s)=\lambda(s) p^{*}(s) \int_{0}^{1} \Psi^{*}(\tau)\left(T f_{x}\right)_{s}(s)(\tau) \Phi(s)(\tau) q(s) d \tau
$$

The natural transversality condition for the period-doubling bifurcation is $\lambda_{s}\left(s_{0}\right) \neq 0$. We now show that this is equivalent to $G_{s}\left(s_{0}\right) \neq 0$, thus establishing regularity.

Proposition 18. The conditions $\lambda_{s}\left(s_{0}\right) \neq 0$ and $G_{s}\left(s_{0}\right) \neq 0$ are equivalent.

Proof. The equations (3.22) are to be considered as identities in $s$; by taking derivatives we obtain

$$
\begin{gathered}
\left(D-T f_{x}\right) v_{s}=\left(T f_{x}\right)_{s} v-\psi_{0} G_{s}, \\
\left(\delta_{0}+\delta_{1}\right) v_{s}=0 \\
\operatorname{Int}_{\phi_{0}} v_{s}=0 .
\end{gathered}
$$

The solution of $(3.22)$ at $s=s_{0}$ is given by $G\left(s_{0}\right)=0, v\left(s_{0}\right)(t)=\Phi\left(s_{0}\right)(t) q_{0}$. Now, at $s=s_{0}(4.10)$ is a linear differential equation for $v_{s}\left(s_{0}\right)(t)$ with solution

$$
v_{s}\left(s_{0}\right)(t)=\Phi\left(s_{0}\right)(t)\left[\zeta+\int_{0}^{t} \Psi^{*}\left(s_{0}\right)(\tau)\left(\left(T f_{x}\right)_{s}\left(s_{0}\right)(\tau) v\left(s_{0}\right)(\tau)-\psi_{0} G_{s}\left(s_{0}\right)\right) d \tau\right]
$$

for some vector $\zeta \in \mathbf{R}^{n}$. Using (4.11) we find

$0=\left(I+\Phi\left(s_{0}\right)(1)\right) \zeta+\Phi\left(s_{0}\right)(1) \int_{0}^{1} \Psi^{*}\left(s_{0}\right)(\tau)\left(\left(T f_{x}\right)_{s}\left(s_{0}\right)(\tau) \Phi\left(s_{0}\right)(\tau) q_{0}-\psi_{0} G_{s}\left(s_{0}\right)\right) d \tau$

This equation in $\zeta$ has a solution if and only if

$$
p^{*}\left(s_{0}\right) \Phi\left(s_{0}\right)(1) \int_{0}^{1} \Psi^{*}\left(s_{0}\right)(\tau)\left(\left(T f_{x}\right)_{s}\left(s_{0}\right)(\tau) \Phi\left(s_{0}\right)(\tau) q_{0}-\psi_{0} G_{s}\left(s_{0}\right)\right) d \tau=0
$$

that is,

$$
p_{0}^{*} \int_{0}^{1} \Psi^{*}\left(s_{0}\right)(\tau)\left(\left(T f_{x}\right)_{s}\left(s_{0}\right)(\tau) \Phi\left(s_{0}\right)(\tau) q_{0} d \tau=\left\langle\psi_{0}, \Psi p_{0}\right\rangle G_{s}\left(s_{0}\right)\right.
$$

By (4.9) this implies

$$
-\left(p_{0}^{*} q_{0}\right) \lambda_{s}\left(s_{0}\right)=\left\langle\psi_{0}, \Psi p_{0}\right\rangle G_{s}\left(s_{0}\right) .
$$

Since $p_{0}^{*} q_{0}$ and $\left\langle\psi_{0}, \Psi p_{0}\right\rangle$ are nonzero, this completes the proof. $\diamond$ 
4.3. Regularity at a torus bifurcation. Again, let $s$ be arclength along the curve of periodic orbits, so that $(x(s)(t), T(s), \alpha(s))$ is a solution of $(2.1),(2.2)$, and (2.3) for all $s$ near the torus bifurcation value $s_{0}$. We assume that $\Phi\left(s_{0}\right)(1)$ has algebraically simple eigenvalues $e^{ \pm i \theta}$. Let $\lambda(s)=\lambda_{1}(s)+i \lambda_{2}(s), p(s)=p_{1}(s)+i p_{2}(s)$, $q(s)=q_{1}(s)+i q_{2}(s)$ be the smooth continuations of the critical multiplier $e^{i \theta}$ and the corresponding left and right eigenvectors. The natural transversality condition is the requirement that $\lambda(s)$ crosses the unit circle in the complex plane transversally, i.e.,

$$
\lambda_{1}\left(s_{0}\right) \lambda_{1 s}\left(s_{0}\right)+\lambda_{2}\left(s_{0}\right) \lambda_{2 s}\left(s_{0}\right) \neq 0 .
$$

Proposition 19. The system consisting of (2.1), (2.2), (2.3), and the conditions

$$
\begin{aligned}
& G_{11}=0, \\
& G_{12}=0, \\
& G_{21}=0, \\
& G_{22}=0,
\end{aligned}
$$

where the $G_{i j}$ are defined in Proposition 17, together form a defining system with full linear rank for the torus bifurcation points of periodic solutions if the natural transversality condition (4.13) is satisfied.

Proof. To prove that the system (2.1), (2.2),(2.3),(4.14) is a defining system with full linear rank we consider the implicit solution $(x(s)(t), T(s), \alpha(s))$ of $(2.1)$, $(2.2),(2.3)$. So $G_{11}, G_{12}, G_{21}, G_{22}$ are functions of $s, \kappa$ only and we have to prove that

$$
\left(\begin{array}{cc}
G_{11 s} & G_{11 \kappa} \\
G_{12 s} & G_{12 \kappa} \\
G_{21 s} & G_{21 \kappa} \\
G_{22 s} & G_{22 \kappa}
\end{array}\right)
$$

has rank 2. Assume that $c_{1}, c_{2} \in \mathbf{R}$ are such that

$$
c_{1} G_{i j s}+c_{2} G_{i j \kappa}=0, \quad(i, j=1,2) .
$$

We start by noting that $p^{H}(s) q(s) \neq 0$ in a neighborhood of $s=s_{0}$. By standard arguments

$$
\left(p^{H} q\right) \lambda_{s}=p^{H} \Phi_{s}(1) q,
$$

where for simplicity of notation we have suppressed the dependence on $s$. To get an expression for $\Phi_{s}(1)$ we start from the identity

$$
\left(D-T f_{x}\right) \Phi \equiv 0
$$

Taking derivatives with respect to $s$ and multiplying with any vector $\zeta \in \mathbf{R}^{n}$ we find

$$
\left(D-T f_{x}\right) \Phi_{s} \zeta=\left(T f_{x}\right)_{s} \Phi \zeta
$$

The solution of this linear differential equation in $\Phi_{s} \zeta$ is

$$
\Phi_{s} \zeta(t)=\Phi(s)(t)\left[\xi+\int_{0}^{t} \Psi^{*}(s)(\tau)\left(T f_{x}\right)_{s}(s)(\tau) \Phi(s)(\tau) \zeta d \tau\right]
$$


where $\xi$ is determined by the initial conditions. Since for $t=0$ we have $\Phi(0)=$ $I, \Phi_{s}(0)=0$, it follows that $\xi=0$. Choosing $\zeta=q$ we obtain from (4.16) that

$$
\left(p^{H} q\right) \lambda_{s}=\lambda p^{H} \int_{0}^{1} \Psi^{*}(s)(\tau)\left(T f_{x}\right)_{s}(s)(\tau) \Phi(s)(\tau) q d \tau .
$$

From (3.31) we infer that

$$
\begin{gathered}
M_{5}\left[\begin{array}{cc}
v_{1 s} & v_{2 s} \\
G_{11 s} & G 12 s \\
G_{21 s} & G_{22 s}
\end{array}\right]=\left[\begin{array}{cc}
\left(T f_{x}\right)_{s} v_{1} & \left(T f_{x}\right)_{s} v_{2} \\
0 & 0 \\
0 & 0 \\
0 & 0
\end{array}\right], \\
M_{5}\left[\begin{array}{cc}
v_{1 \kappa} & v_{2 \kappa} \\
G_{11 \kappa} & G_{12 \kappa} \\
G_{21 \kappa} & G_{22 \kappa}
\end{array}\right]=\left[\begin{array}{cc}
0 & 0 \\
2 v_{1}(1) & 2 v_{2}(1) \\
0 & 0 \\
0 & 0
\end{array}\right] .
\end{gathered}
$$

Combining (4.15),(4.18) and (4.19) we obtain

(4.20) $M_{5}\left[\begin{array}{cc}c_{1} v_{1 s}+c_{2} v_{1 \kappa} & c_{1} v_{2 s}+c_{2} v_{2 \kappa} \\ 0 & 0 \\ 0 & 0\end{array}\right]=\left[\begin{array}{cc}c_{1}\left(T f_{x}\right)_{s} v_{1} & c_{1}\left(T f_{x}\right)_{s} v_{2} \\ 2 c_{2} v_{1}(1) & 2 c_{2} v_{2}(1) \\ 0 & 0 \\ 0 & 0\end{array}\right]$

Hence

$$
\left(\begin{array}{c}
c_{1}\left(T f_{x}\right)_{s} v_{1} \\
2 c_{2} v_{1}(1)
\end{array}\right),\left(\begin{array}{c}
c_{1}\left(T f_{x}\right)_{s} v_{2} \\
2 c_{2} v_{2}(1)
\end{array}\right)
$$

are both in the range of (3.24). As an essential step in the proof of Proposition 15 it was shown that this implies

$$
\begin{aligned}
& c_{1} L\left(\left(T f_{x}\right)_{s} v_{1}\right)=2 c_{2} p^{H} v_{1}(1), \\
& c_{1} L\left(\left(T f_{x}\right)_{s} v_{2}\right)=2 c_{2} p^{H} v_{2}(1),
\end{aligned}
$$

where the linear operator $L$ is defined in (3.25). Since $v_{1}, v_{2}$ are in the kernel of (3.24) we have

$$
v_{1}(\tau)=\Phi(\tau) v_{1}(0), v_{2}(\tau)=\Phi(\tau) v_{2}(0) .
$$

Combining the last four formulae we find

$$
c_{1} L\left(\left(T f_{x}\right)_{s} \Phi q\right)=2 c_{2} p^{H} \Phi(1) q=2 c_{2} e^{i \theta}\left(p^{H} q\right) .
$$

Now,

$$
\begin{aligned}
L\left(\left(T f_{x}\right)_{s} \Phi q\right)= & -2 \kappa e^{i \theta} \int_{0}^{1} p^{H} \Psi^{*}(\tau)\left(T f_{x}\right)_{s} \Phi(\tau) q d \tau+e^{2 i \theta} \int_{0}^{2} p^{H} \Psi^{*}(\tau)\left(T f_{x}\right)_{s} \Phi(\tau) q d \tau \\
= & e^{i \theta}(\cos \theta+i \sin \theta-2 \cos \theta) \int_{0}^{1} p^{H} \Psi^{*}(\tau)\left(T f_{x}\right)_{s} \Phi(\tau) q d \tau \\
& +e^{2 i \theta} \int_{0}^{1} p^{H} \Psi^{*}(1+\tau)\left(T f_{x}\right)_{s} \Phi(1+\tau) q d \tau
\end{aligned}
$$


Also,

$$
p^{H} \Psi^{*}(1+\tau)=(\Psi(1+\tau) p)^{H}=(\Psi(\tau) \Psi(1) p)^{H}=p^{H} \Phi^{-1}(1) \Psi^{*}(\tau)=e^{-i \theta} p^{H} \Psi^{*}(\tau)
$$

and

$$
\Phi(1+\tau) q=\Phi(\tau) \Phi(1) q=e^{i \theta} \Phi(\tau) q
$$

Hence

$$
L\left(\left(T f_{x}\right)_{s} \Phi q\right)=e^{i \theta} 2 i \sin \theta \int_{0}^{1} p^{H} \Psi^{*}(\tau)\left(T f_{x}\right)_{s} \Phi(\tau) q d \tau .
$$

By (4.17) this implies

$$
L\left(\left(T f_{x}\right)_{s} \Phi q\right)=2 i \sin \theta\left(p^{H} q\right) \lambda_{s} .
$$

Using (4.21) we further obtain

$$
2 i c_{1} \sin \theta\left(p^{H} q\right) \lambda_{s}=2 c_{2} e^{i \theta}\left(p^{H} q\right) .
$$

Dividing by $2\left(p^{H} q\right)$ we obtain

$$
\left(-\sin \theta \lambda_{2 s}+i \sin \theta \lambda_{1 s}\right) c_{1}=(\cos \theta+i \sin \theta) c_{2} .
$$

Taking real and imaginary parts of this complex equality we find

$$
\left(\begin{array}{cc}
-\sin \theta \lambda_{2 s} & -\cos \theta \\
\sin \theta \lambda_{1 s} & -\sin \theta
\end{array}\right)\left(\begin{array}{l}
c_{1} \\
c_{2}
\end{array}\right)=\left(\begin{array}{l}
0 \\
0
\end{array}\right)
$$

The determinant of the $2 \times 2$ matrix in this expression is equal to

$$
\sin \theta\left(\cos \theta \lambda_{1 s}+\sin \theta \lambda_{2 s}\right)=\sin \theta\left(\lambda_{1} \lambda_{1 s}+\lambda_{2} \lambda_{2 s}\right) .
$$

By (4.13) and $\sin \theta \neq 0$ this implies that $c_{1}=c_{2}=0$, which completes the proof. $\diamond$

5. Computational issues. In this section we discuss computational issues related to the implementation of our defining systems; namely the computation of the derivatives of the test functionals with respect to the unknowns of the system, $x(t), \alpha, T$, as well as the problem of adapting the defining systems along the bifurcation branch. We also explicitly show the BVPs that must be solved.

5.1. Fold bifurcation. Proposition 9 implies that locally, near a fold bifurcation of periodic solutions, the system consisting of (2.1), (2.2), (2.3) and

$$
G=0,
$$

defines the set of fold bifurcation points in $(x(\cdot), T, \alpha)$-space; here $G$ is defined by (3.14). Under natural nondegeneracy and transversality conditions, the regularity of this system was proved in $\$ 4.1$.

We need the derivatives of $G$ with respect to the unknowns of the system, i.e., with respect to $x(\cdot), \alpha, T$.

Denoting by $z$ any component of $\alpha$ or $T$ we infer from (3.14) that

$$
N_{1}\left(\begin{array}{c}
v_{z} \\
S_{z} \\
G_{z}
\end{array}\right)=\left(\begin{array}{c}
{\left[T f_{x}(x(t), \alpha]_{z} v+[f(x(t), \alpha)]_{z} S\right.} \\
0 \\
\operatorname{Int}_{[f(x(\cdot), \alpha)]_{z}} v \\
0
\end{array}\right) .
$$


Numerically we solve a discretized version of this equation, say

$$
N_{1}^{d}\left(\begin{array}{c}
v_{z} \\
S_{z} \\
G_{z}
\end{array}\right)=\left(\begin{array}{c}
\left(\left[T f_{x}(x(t), \alpha]_{z} v+[f(x(t), \alpha)]_{z} S\right)_{d}\right. \\
0 \\
\left(\operatorname{Int}_{[f(x(\cdot), \alpha)]_{z}} v\right)_{d} \\
0
\end{array}\right) .
$$

where $N_{1}^{d}$ is the discretized version of $N_{1}$, i.e., a large square matrix with a structure that can be efficiently factorized, for example, as in AUTo.

Note that a large number of linear systems having the same structured matrix $N_{1}^{d}$ must be solved. Moreover, all right hand sides are known before the factorization. Thus the solution be done in a single factorization process, without storing the factors.

$\left(N_{1}^{d}\right)^{T}$ has a block structure that is very similar to $N_{1}^{d}$. If an efficient solution strategy for $\left(N_{1}^{d}\right)^{T}$ is also developed, then it is possible to avoid solving (5.3) for all relevant $z$. Instead, a single system with $\left(N_{1}^{d}\right)^{T}$ is to be solved. In transposed form it is given by

$$
\left(w_{1}^{*}, w_{2}^{*}, w_{3}, w_{4}\right) N_{1}^{d}=(0,0,1) .
$$

Combining (5.3) and (5.4) we find

$$
G_{z}=w_{1}^{*}\left(\left[T f_{x}(x(t), \alpha]_{z} v+[f(x(t), \alpha)]_{z} S\right)_{d}+w_{3}\left(\operatorname{Int}_{[f(x(\cdot), \alpha)]_{z}} v\right)_{d} .\right.
$$

Notice that (3.14) is equivalent to the system

$$
\left\{\begin{aligned}
v^{\prime}(t)-T f_{x}(x(t), \alpha) v(t)-S f(x(t), \alpha)+G w_{01}(t) & =0 \\
v(1)-v(0)+G w_{02} & =0 \\
\int_{0}^{1} v^{*}(\tau) f(x(\tau), \alpha) d \tau+G w_{03} & =0 \\
\int_{0}^{1} v^{*}(\tau) v_{01}(\tau) d \tau+S v_{02} & =1
\end{aligned}\right.
$$

while (3.15) can be explicitly written as

$$
\left\{\begin{aligned}
w^{\prime}(t)+T f_{x}^{*}(x(t), \alpha) w(t)-R f(x(t), \alpha)+H v_{11}(t) & =0 \\
w(1)-w(0)+H v_{12} & =0 \\
\int_{0}^{1} w^{*}(\tau) f(x(\tau), \alpha) d \tau+H v_{13} & =0 \\
\int_{0}^{1} w^{*}(\tau) w_{11}(\tau) d \tau+R w_{12} & =-1
\end{aligned}\right.
$$

Discretizations of these systems, for example by orthogonal collocation, result in linearized Newton systems having the same sparsity as the linear systems arising from (2.5). They can therefore be solved using the same numerical linear algebra algorithms.

In practice we need to adapt the auxiliary variables (i.e., $w_{01}, w_{02}, w_{03}, v_{01}, v_{02}, v_{11}$, $v_{12}, v_{13}, w_{11}$, and $w_{12}$ ) along a computed branch of fold bifurcations of periodic orbits. For the bordering rows in $N_{1}$ and $N_{2}$ the natural choice is to take the kernel vectors of $M_{1}$ and $M_{2}$ respectively, at a previously computed solution point. These kernel vectors are obtained as a byproduct of solving (5.5) and (5.6). For the column bordering of $N_{1}$ we need a vector not in the range of $M_{1}$. By Proposition 6 , a possible choice is

$$
\left(\begin{array}{l}
w_{01} \\
w_{02} \\
w_{03}
\end{array}\right)=\left(\begin{array}{c}
\Psi p_{0} \\
0 \\
0
\end{array}\right),
$$


which by Proposition 7 can be derived from the solution of (5.6). Finally, a bordering column for $\mathrm{N}_{2}$ is given in Proposition 8:

$$
\left(\begin{array}{l}
v_{11} \\
v_{12} \\
v_{13}
\end{array}\right)=\left(\begin{array}{l}
0 \\
0 \\
1
\end{array}\right)
$$

Therefore, problems (5.5) and (5.6) actually take the following simplified forms:

$$
\left\{\begin{aligned}
v^{\prime}(t)-T f_{x}(x(t), \alpha) v(t)-S f(x(t), \alpha)+G w_{01}(t) & =0 \\
v(1)-v(0) & =0 \\
\int_{0}^{1} v^{*}(\tau) f(x(\tau), \alpha) d \tau & =0 \\
\int_{0}^{1} v^{*}(\tau) v_{01}(\tau) d \tau+S & =1
\end{aligned}\right.
$$

and

$$
\left\{\begin{aligned}
w^{\prime}(t)+T f_{x}^{*}(x(t), \alpha) w(t)-R f(x(t), \alpha) & =0 \\
w(1)-w(0) & =0 \\
\int_{0}^{1} w^{*}(\tau) f(x(\tau), \alpha) d \tau+H & =0 \\
\int_{0}^{1} w^{*}(\tau) w_{11}(\tau) d \tau & =-1
\end{aligned}\right.
$$

5.2. Period-doubling. By Proposition 14 period-doubling bifurcations are determined by (2.1), (2.2), (2.3), and the condition $G=0$, where $G$ is given by (3.22); assuming the conditions $\left\langle\phi_{0}, \Phi q_{0}\right\rangle \neq 0,\left\langle\psi_{0}, \Psi p_{0}\right\rangle \neq 0$ hold. To solve such systems numerically, we need the derivatives of $G$ with respect to the unknowns of the system, i.e., with respect to $x(t), \alpha, T$. These can be approximated by finite differences, using (3.22). As in the fold case, they can be obtained exactly by solving an "adjoint problem" to (3.22). In this case the adjoint problem is (3.23).

Proposition 20. Let $z$ denote a component of the problem parameter vector $\alpha$, or let $z$ denote the period $T$, on both of which the quantity $G$ in $(3.22)$ depends. Let $v$ and $w$ be obtained from $(3.22)$ and $(3.23)$, respectively. Then the derivative of $G$ with respect to $z$ can be written as

$$
G_{z}=-\int_{0}^{1} w^{*}(\tau)\left[T f_{x}(x(\tau), \alpha)\right]_{z} v(\tau) d \tau
$$

while the linear part of the variation of $G$ with respect to $x \mapsto x+\delta x$ is given by

$$
\left.\delta G=-\int_{0}^{1} w^{*}(\tau) T f_{x x}(x(\tau), \alpha)\right) v(\tau)(\delta x)(\tau) d \tau
$$

Proof. By differentiating (3.22) we obtain

$$
M_{1}\left(\begin{array}{c}
v_{z} \\
G_{z}
\end{array}\right)=\left(\begin{array}{c}
{\left[T f_{x}(x(t), \alpha)\right]_{z} v} \\
0 \\
0
\end{array}\right) .
$$

Multiplying the first equation in (5.9) from the left with $w^{*}$, integrating over the interval $[0,1]$ and using the third equation in (3.23) we get

$$
\begin{aligned}
& \int_{0}^{1} w^{*}(\tau) v_{z}^{\prime}(\tau) d \tau-\int_{0}^{1} w^{*}(\tau) T f_{x}(x(\tau), \alpha) v_{z}(\tau) d \tau-G_{z}= \\
& \int_{0}^{1} w^{*}(\tau)\left[T f_{x}(x(\tau), \alpha)\right]_{z} v(\tau) d \tau
\end{aligned}
$$


By partial integration of the first term in this expression and using the second equations in (3.23) and (5.9) we obtain

$$
\begin{aligned}
& -\int_{0}^{1} v_{z}^{*}(\tau) w^{\prime}(\tau) d \tau-\int_{0}^{1} v_{z}^{*}(\tau) T f_{x}^{*}(x(\tau), \alpha) w(\tau) d \tau-G_{z}= \\
& \int_{0}^{1} w^{*}(\tau)\left[T f_{x}(x(\tau), \alpha)\right]_{z} v(\tau) d \tau
\end{aligned}
$$

Using the first equation in (3.23) we get

$$
-\int_{0}^{1} v_{z}^{*}(\tau)\left(-\phi_{0}(\tau) H\right) d \tau-G_{z}=\int_{0}^{1} w^{*}(\tau)\left[T f_{x}(x(\tau), \alpha)\right]_{z} v(\tau) d \tau
$$

By the last equation in (5.9) the first part of the Proposition follows.

The linear parts of the variations of $G$ and $v$ under variation of $x$ satisfies

$$
M_{1}\left(\begin{array}{c}
\delta v \\
\delta G
\end{array}\right)=\left(\begin{array}{c}
T f_{x x}(x(t), \alpha) v \delta x \\
0 \\
0
\end{array}\right)
$$

Similar to the derivation above, this implies the second part of the Proposition. $\diamond$

Notice that (3.22) is equivalent to the system

$$
\left\{\begin{array}{cl}
v^{\prime}(t)-T f_{x}(x(t), \alpha) v(t)+G \psi_{0}(t) & =0 \\
v(0)+v(1) & =0 \\
\int_{0}^{1} \phi_{0}^{*}(\tau) v(\tau) d \tau & =1
\end{array}\right.
$$

while (3.23) can be explicitly written as

$$
\left\{\begin{array}{cl}
w^{\prime}(t)+T f_{x}^{*}(x(t), \alpha) w(t)+H \phi_{0}(t) & =0 \\
w(0)+w(1) & =0 \\
\int_{0}^{1} \psi_{0}^{*}(\tau) w(\tau) d \tau & =
\end{array}\right.
$$

Discretizations of these systems, for example by orthogonal collocation, result in linearized Newton systems having the same sparsity as the linear systems arising from (2.5). They can therefore be solved using the same numerical linear algebra algorithms.

The natural choice for starting values of $\phi_{0}, \psi_{0}$ is

$$
\phi_{0}(t)=\Phi(t) q_{0}, \quad \psi_{0}(t)=\Psi(t) p_{0} .
$$

In a continuation context, it is necessary to regularly update $\phi_{0}$ and $\psi_{0}$. Specifically, $v$ obtained from (3.22) can be used to update $\phi_{0}$, and $w$ obtained from (3.23) can be used to update $\psi_{0}$. Indeed, after convergence to a period-doubling bifurcation, $v$ spans the kernel of

$$
\left(\begin{array}{c}
D-T f_{x}(x(t), \alpha) \\
\delta_{0}+\delta_{1}
\end{array}\right)
$$

and similarly, $w$ spans the kernel of

$$
\left(\begin{array}{c}
D+T f_{x}^{*}(x(t), \alpha) \\
\delta_{0}+\delta_{1}
\end{array}\right)
$$


5.3. Torus bifurcation. We have proved in Proposition 17 that the matrix equation $G=0$ can be used to continue numerically curves of torus bifurcation points. Some issues require further attention.

First of all, we mention that the BVP for $G$ is defined on the interval $[0,2]$ and that 3-point boundary conditions are involved (at $t=0,1$, and 2 ).

To solve the system (2.1), (2.2), (2.3), (4.14) efficiently by a Newton-like method, one needs the derivatives $G_{i j z}$, where $z$ is $T$ or a component of $\alpha$. From (3.31) we infer that

$$
M_{5}\left(\begin{array}{cc}
v_{1 z} & v_{2 z} \\
G_{11 z} & G_{12 z} \\
G_{21 z} & G_{22 z}
\end{array}\right)=\left(\begin{array}{cc}
{\left[T f_{x}(x(t), \alpha]_{z} v_{1}\right.} & {\left[T f_{x}(x(t), \alpha)\right]_{z} v_{2}} \\
0 & 0 \\
0 & 0 \\
0 & 0
\end{array}\right)
$$

One also needs the derivatives with respect to $\kappa$; for this we find

$$
M_{5}\left(\begin{array}{cc}
v_{1 \kappa} & v_{2 \kappa} \\
G_{11 \kappa} & G_{12 \kappa} \\
G_{21 \kappa} & G_{22 \kappa}
\end{array}\right)=\left(\begin{array}{cc}
0 & 0 \\
2 v_{1}(1) & 2 v_{2}(1) \\
0 & 0 \\
0 & 0
\end{array}\right)
$$

Numerically we solve the discretized versions of these equations, say

$$
M_{5}^{d}\left(\begin{array}{cc}
v_{1 z} & v_{2 z} \\
G_{11 z} & G_{12 z} \\
G_{21 z} & G_{22 z}
\end{array}\right)=\left(\begin{array}{cc}
{\left[T f_{x}(x(t), \alpha]_{z} v_{1}\right.} & {\left[T f_{x}(x(t), \alpha)\right]_{z} v_{2}} \\
0 & 0 \\
0 & 0 \\
0 & 0
\end{array}\right)
$$

One also needs the derivatives with respect to $\kappa$; for this we find

$$
M_{5}^{d}\left(\begin{array}{cc}
v_{1 \kappa} & v_{2 \kappa} \\
G_{11 \kappa} & G_{12 \kappa} \\
G_{21 \kappa} & G_{22 \kappa}
\end{array}\right)=\left(\begin{array}{cc}
0 & 0 \\
2 v_{1}(1) & 2 v_{2}(1) \\
0 & 0 \\
0 & 0
\end{array}\right)
$$

where $M_{5}^{d}$ is the discretized version of $M_{5}$, i.e., a large square matrix of the same structure as that factored efficiently in AUTO.

We again note that a large number of linear systems with the same structured matrix $M_{5}^{d}$ has to be solved. All right hand sides are known when the factorization is done. Thus the solution of all systems can be done during a single factorization process of $M_{5}^{d}$ without storing the factors.

$\left(M_{5}^{d}\right)^{*}$ has a block structure that is very similar to that of $M_{5}^{d}$. If an efficient solution strategy for $\left(M_{5}^{d}\right)^{*}$ is also developed, then it is possible to avoid solving (5.14) for all relevant $z$ and (5.15). Instead, a single system with $\left(M_{5}^{d}\right)^{*}$ is to be solved. In transposed form it is given by

$$
\left(\begin{array}{cccc}
w_{1}^{1 *} & w_{1}^{2 *} & G_{11} & G_{12} \\
w_{2}^{1 *} & w_{2}^{2 *} & G_{21} & G_{22}
\end{array}\right) M_{5}^{d}=\left(\begin{array}{cccc}
0 & 0 & 1 & 0 \\
0 & 0 & 0 & 1
\end{array}\right) .
$$

Combining (5.14) and (5.16) we find

$$
\left(\begin{array}{ll}
G_{11 z} & G_{12 z} \\
G_{21 z} & G_{22 z}
\end{array}\right)=\left(\begin{array}{cc}
w_{1}^{1 *}\left[T f_{x}(x(t), \alpha)\right]_{z} v_{1} & w_{1}^{1 *}\left[T f_{x}(x(t), \alpha)\right]_{z} v_{2} \\
w_{2}^{1 *}\left[T f_{x}(x(t), \alpha)\right]_{z} v_{1} & w_{2}^{1 *}\left[T f_{x}(x(t), \alpha)\right]_{z} v_{2}
\end{array}\right)
$$


if $z$ is $T$ or one of the components of $x, \alpha$. For $\kappa$ we find

$$
\left(\begin{array}{ll}
G_{11 \kappa} & G_{12 \kappa} \\
G_{21 \kappa} & G_{22 \kappa}
\end{array}\right)=\left(\begin{array}{ll}
2 w_{1}^{2 *} v_{1}(1) & 2 w_{1}^{2 *} v_{2}(1) \\
2 w_{2}^{2 *} v_{1}(1) & 2 w_{2}^{2 *} v_{2}(1)
\end{array}\right) .
$$

Next notice that (3.31) is equivalent to the system

$$
\left\{\begin{array}{cc}
v_{1}^{\prime}-T f_{x}(x(t), \alpha) v_{1}+G_{11} \psi_{0}+G_{21} \psi_{1} & =0 \\
v_{2}^{\prime}-T f_{x}(x(t), \alpha) v_{2}+G_{12} \psi_{0}+G_{22} \psi_{1} & =0 \\
v_{1}(0)-2 \kappa v_{1}(1)+v_{1}(2) & =0 \\
v_{2}(0)-2 \kappa v_{2}(1)+v_{2}(2) & =0 \\
\int_{0}^{2} \phi_{0}^{*}(\tau) v_{1}(\tau) d \tau & =1 \\
\int_{0}^{2} \phi_{1}^{*}(\tau) v_{2}(\tau) d \tau & =0 \\
\int_{0}^{2} \phi_{0}^{*}(\tau) v_{1}(\tau) d \tau & =0 \\
\int_{0}^{2} \phi_{1}^{*}(\tau) v_{2}(\tau) d \tau & =1,
\end{array}\right.
$$

while (3.32) can be explicitly written as

$$
\left\{\begin{array}{ccc}
w_{1}^{\prime}+T f_{x}^{*}(x(t), \alpha) w_{1}+H_{11} \phi_{0}+H_{21} \phi_{1} & = & 0 \\
w_{2}^{\prime}+T f_{x}^{*}(x(t), \alpha) w_{2}+H_{12} \phi_{0}+H_{22} \phi_{1} & = & 0 \\
w_{1}(0)-2 \kappa w_{1}(1)+w_{1}(2) & = & 0 \\
w_{2}(0)-2 \kappa w_{2}(1)+w_{2}(2) & = & 0 \\
\int_{0}^{2} \psi_{0}^{*}(\tau) w_{1}(\tau) d \tau & = & -1, \\
\int_{0}^{2} \psi_{1}^{*}(\tau) w_{2}(\tau) d \tau & = & 0 \\
\int_{0}^{2} \psi_{0}^{*}(\tau) w_{1}(\tau) d \tau & = & 0 \\
\int_{0}^{2} \psi_{1}^{*}(\tau) w_{2}(\tau) d \tau & = & -1 .
\end{array}\right.
$$

Discretizations of these systems, for example by orthogonal collocation, result in linearized Newton systems having the same sparsity as the linear systems arising from (2.5). They can therefore be solved using the same numerical linear algebra algorithms.

In a continuation context the vector-functions $\phi_{0}, \phi_{1}, \psi_{0}, \psi_{1}$ should be updated. This can be done by solving both (5.17) and (5.18). Indeed, $v_{1}, v_{2}$ span the twodimensional space in which $\phi_{0}, \phi_{1}$ should be chosen and $w_{1}, w_{2}$ similarly span the space in which $\psi_{0}, \psi_{1}$ should be chosen (some orthogonalization and scaling may be appropriate).

Finally recall that we compute the torus bifurcation points by using essentially an overdetermined system. This should necessitate some changes in the elimination strategy when solving the linear systems.

6. Numerical example. In this section we illustrate the proposed techniques on a test example: a simple feedback control system of Lur'e type:

$$
\left\{\begin{array}{l}
\dot{x}_{1}=x_{2}, \\
\dot{x}_{2}=x_{3} \\
\dot{x}_{3}=-\alpha x_{3}-\beta x_{2}-x_{1}+x_{1}^{2},
\end{array}\right.
$$

where $\alpha$ and $\beta$ are positive parameters. It is well known (see, for example [15], Section 5.4) that the equilibrium $x_{1}=x_{2}=x_{3}=0$ of (6.1) exhibits at

$$
\alpha_{0}=\frac{1}{\beta}
$$


a supercritical Hopf bifurcation generating a stable periodic solution that exists for $\alpha<\alpha_{0}$. This periodic solution undergoes a supercritical period-doubling bifurcation at $\alpha_{1}=0.630302 \ldots$

A discretized continuation problem (2.1), (2.2), and (2.3) for the periodic solution is coded for the matla collocation with piecewise polynomials is used, which is similar to one implemented in AUTO and is characterized by the number NTST of mesh points and the number NCOL of collocation points. At each computed point in the solution curve, a discrete version of (5.10) is set up and solved. This gives a value of the test function $G$ to detect period doubling. A constant bordering function $\psi_{0}$ is used, while the computed approximation to $v$ is used to update the bordering function $\phi_{0}$. Figures 1 and 2 are produced with NTST $=10$ and NCOL $=4$.

Figure 1 shows the behavior of $G$ as a function of $\alpha$ for $\beta=1$. For this value of $\beta$, Hopf bifurcation occurs at $\alpha_{0}=1$. In the same figure, the function $\mu_{1}+1$ is plotted, where $\mu_{1}$ is a nontrivial Floquet multiplier of the periodic solution for which $\mu_{1}\left(\alpha_{1}\right)=-1$. The multipliers are computed via a specially adapted elimination algorithm from AUTo. Clearly, $G$ vanishes together with $\mu_{1}+1$. Moreover, close examination of numerical data gives the above bifurcation value $\alpha_{1}$ with 7 correct decimal places. Figure 2 shows a family of computed profiles $v(t)$ along the solution

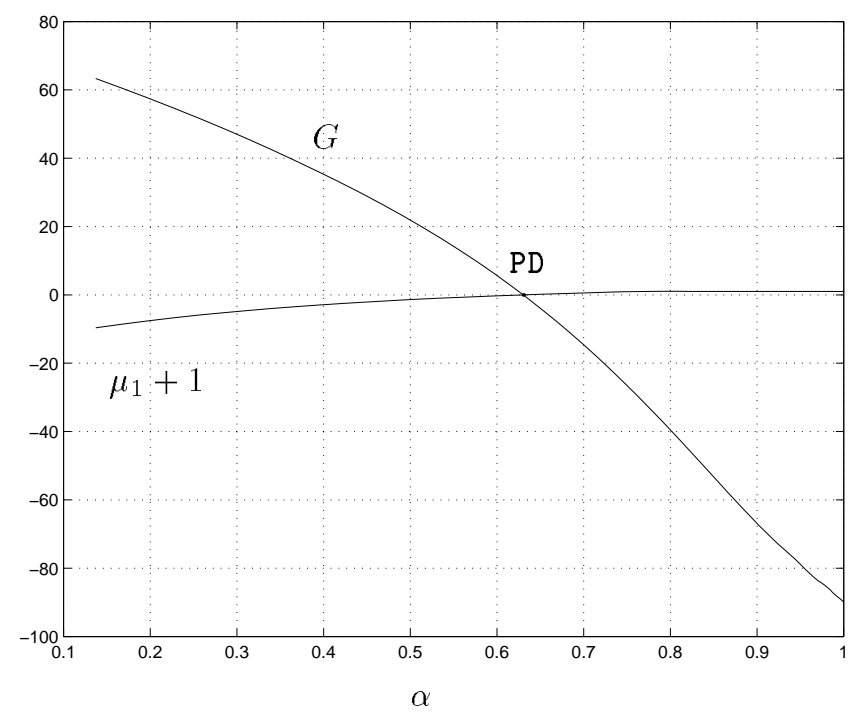

FIG. 1. Test function $G(\alpha)$ and $\mu_{1}(\alpha)+1$ for $\beta=1$.

curve. A dashed solution corresponds to the bifurcation parameter value $\alpha_{1}$. Finally, Figure 3 presents a two-parameter continuation of the period-doubling bifurcation curve, which is closed. The continuation is started at one of the PD points in the one-parameter path of periodic solutions discussed above.

Let us briefly address an important question of comparison of the proposed method to continue the period doubling bifurcation and the algorithm based on the 


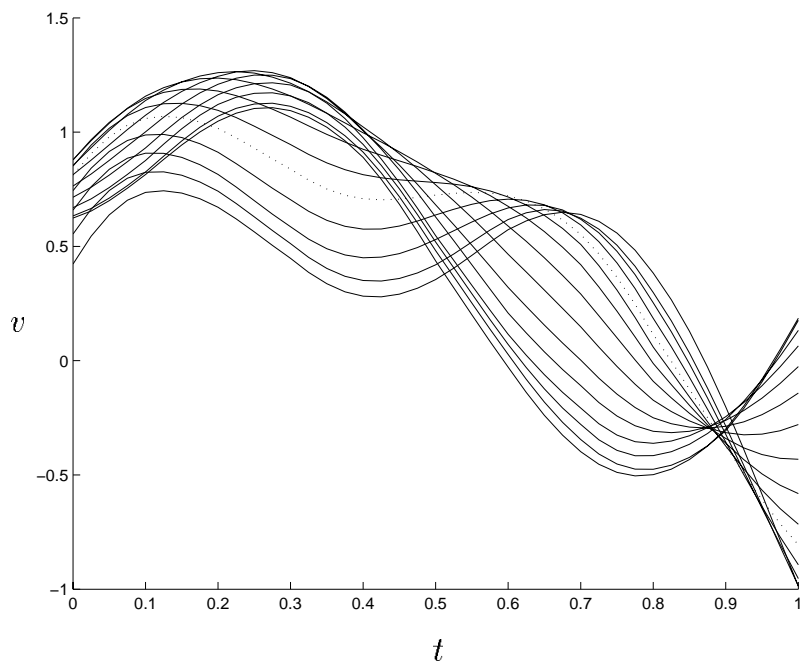

Fig. 2. Solutions $v(t)$ at different $\alpha$-values for $\beta=1$.

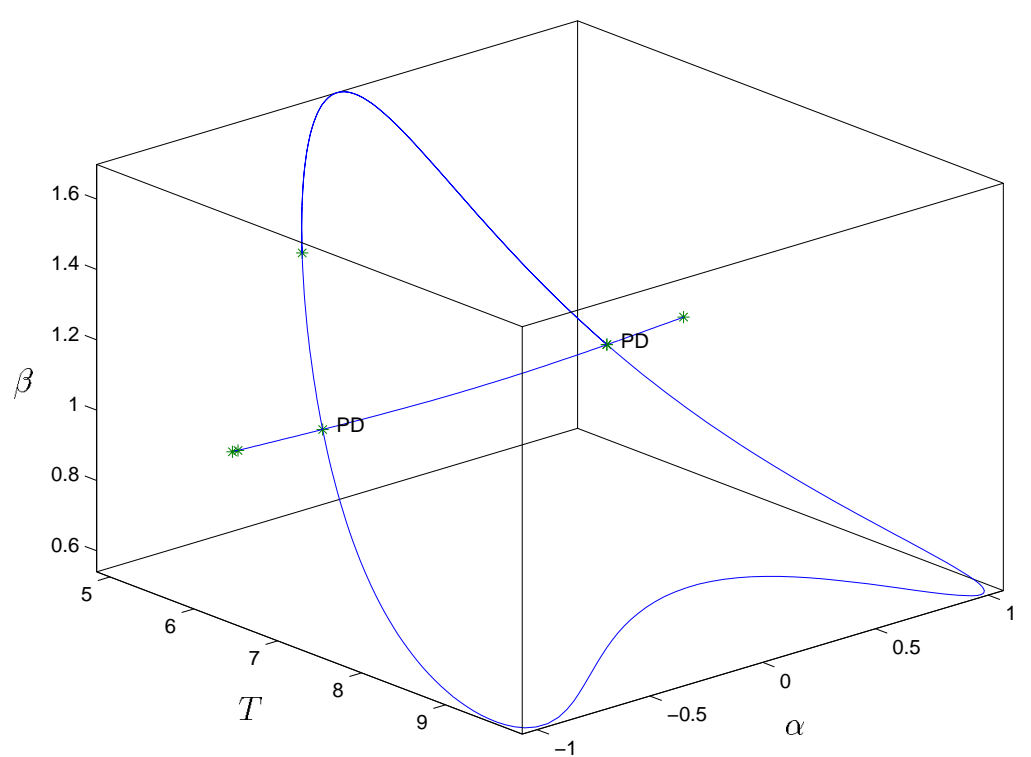

FIG. 3. Cycle and period-doubling branches.

fully extended system, i.e. (2.1), (2.2), and (2.3), augmented by

$$
\left\{\begin{array}{cl}
v^{\prime}(t)-T f_{x}(x(t), \alpha) v(t) & =0 \\
v(0)+v(1) & =0, \\
\int_{0}^{1} \phi_{0}^{*}(\tau) v(\tau) d \tau & =1,
\end{array}\right.
$$

that is implemented in AUTO. The corresponding discretized system is nearly twice the size as the discretized minimally extended system composed of (2.1), (2.2), (2.3), and $G=0$, where $G$ is to be computed from (5.10). However, one has to solve an 
extra BVP (5.11) to calculate the Jacobian matrix of the discretized bordered system. To make a comparison, both methods were similarly implemented using the standard sparse matrix solver in the Continuation Toolbox [17], and tested for different number of mesh and collocation points. The following table shows the execution times required by the two methods to compute on a $350 \mathrm{Mhz}$ PC the same number (300) of points along the period-doubling curve shown in Figure 3 .

\begin{tabular}{|c|c|c|c|}
\hline NTST & NCOL & minimally extended system & fully extended system \\
\hline 10 & 4 & $101,8 \mathrm{~s}$ & $122,3 \mathrm{~s}$ \\
10 & 5 & $134,9 \mathrm{~s}$ & $159,4 \mathrm{~s}$ \\
20 & 4 & $269,9 \mathrm{~s}$ & $358,6 \mathrm{~s}$ \\
20 & 5 & $371,9 \mathrm{~s}$ & $558,2 \mathrm{~s}$ \\
30 & 4 & $529,8 \mathrm{~s}$ & $808,0 \mathrm{~s}$ \\
30 & 5 & $751,0 \mathrm{~s}$ & $1260,3 \mathrm{~s}$ \\
40 & 4 & $886,0 \mathrm{~s}$ & $1528,8 \mathrm{~s}$ \\
40 & 5 & $1376,8 \mathrm{~s}$ & $2528,6 \mathrm{~s}$ \\
\hline
\end{tabular}

Clearly the bordered system is indeed faster and the difference gets bigger when the number of mesh and collocation points increases. More extensive comparisons will be reported elsewhere.

\section{REFERENCES}

[1] E. L. Allgower, K. Georg, Numerical Path Following, Handbook of Numerical Analysis 5, P. G. Ciarlet, J. L. Lions, eds., North Holland Publishing, 1996.

[2] U. M. Ascher, J. Christiansen and R. D. Russell, A collocation solver for mixed order systems of boundary value problems, Math. Comp. 33 (1979) 659-679.

[3] W. J. Beyn, A. Champneys, E. J. Doedel, W. Govaerts, B. Sandstede, Y. A. Kuznetsov, Numerical Continuation and Normalization, Chapter 4 in: Handbook of Dynamical Systems III: Towards Applications, B. Fiedler, ed., Elsevier, 2001.

[4] C. De Boor and B. Swartz, Collocation at Gaussian points, SIAM J. Numer. Anal. 10 (1973) $582-606$.

[5] E. J. Doedel, A. R. Champneys, T. F. Fairgrieve, Yu. A. Kuznetsov, B. Sandstede and X. J. Wang, AUTO97 : Continuation and Bifurcation Software for Ordinary Differential Equations (with HomCont), Concordia University, Montreal, Canada 1997. (ftp.cs.concordia.ca/pub/doedel/auto).

[6] E. J. Doedel, A. D. Jepson, H. B. Keller, Numerical methods for Hopf bifurcation and continuation of periodic solution paths, in: Computing Methods in Applied Sciences and Engineering VI, R. Glowinski, J. L. Lions, eds., North Holland, Amsterdam, 1984, 127-136.

[7] E. J. Doedel, H. B. Keller, J. P. Kernévez, Numerical analysis and control of bifurcation problems: Part I, Int. J. Bifurcation \& Chaos 1 (1991) 493-520.

[8] E. J. Doedel, H. B. Keller, J. P. Kernévez, Numerical analysis and cont rol of bifurcation problems: Part II, Int. J. Bifurcation \& Chaos 1 (1991) 745-772.

[9] T. F. Fairgrieve, PhD Thesis, University of Toronto, 1994.

[10] W. Govaerts, Yu.A. Kuznetsov, and B. Sijnave, Implementation of Hopf and double Hopf continuation using bordering methods. ACM Trans. Math. Software 24 (1998), 418-436.

[11] W. Govaerts, Numerical Methods for Bifurcations of Dynamical Equilibria, SIAM, 2000.

[12] J. Guckenheimer and P. Holmes, Nonlinear Oscillations, Dynamical Systems, and Bifurcations of Vector Fields, Applied Mathematical Sciences 42, Springer Verlag 1983.

[13] A. D. Jepson, PhD Thesis, Applied Mathematics, Caltech, 1981.

[14] H. B. Keller, Numerical solution of bifurcation and nonlinear eigenvalue problems, in: Applications of Bifurcation Theory, P. H. Rabinowitz, ed., Academic Press, 1977, 359-384.

[15] Yu. A. Kuznetsov, Elements of Applied Bifurcation Theory, Second Edition, Springer Verlag, 1998.

[16] Yu. A. Kuznetsov and V. V. Levitin, CONTENT : A multiplatform environment for analyzing dynamical systems, Dynamical Systems Laboratory, CWI, Amsterdam 1995-1997 (ftp.cri.nl/pub/content). 
[17] Yu. A. Kuznetsov, W. Mestrom and A.M. Riet, A Continuation Toolbox in MatLab, Mathematical Institute, Utrecht University, 2001 (ㅂ․ math.uu.nl/people/kuznet/cm).

[18] G. Moore and A. Spence, The calculation of turning points of nonlinear equations, SIAM J. Numer. Anal. 17 (1980) 567-576.

[19] R. D. Russell and J. Christiansen, Adaptive mesh selection strategies for solving boundary value problems, SIAM J. Numer. Anal. 15 (1978) 59-80.

[20] R. Seydel, Numerical computation of branch points in nonlinear equations, Numer. Math. 33 (1979) 339-352. 